\title{
Review Article \\ Impact of Cosmic-Ray Physics on Dark Matter Indirect Searches
}

\author{
Daniele Gaggero ${ }^{1}$ and Mauro Valli $\mathbb{D}^{2}$ \\ ${ }^{1}$ GRAPPA Institute, Institute of Physics, University of Amsterdam, 1098 XH Amsterdam, Netherlands \\ ${ }^{2}$ INFN, Sezione di Roma, P.le A. Moro 2, 00185 Roma, Italy \\ Correspondence should be addressed to Mauro Valli; mauro.valli@romal.infn.it
}

Received 2 February 2018; Accepted 30 April 2018; Published 17 December 2018

Academic Editor: Farinaldo Queiroz

Copyright (C) 2018 Daniele Gaggero and Mauro Valli. This is an open access article distributed under the Creative Commons Attribution License, which permits unrestricted use, distribution, and reproduction in any medium, provided the original work is properly cited. The publication of this article was funded by SCOAP ${ }^{3}$.

\begin{abstract}
The quest for the elusive dark matter (DM) that permeates the Universe (and in general the search for signatures of physics beyond the Standard Model at astronomical scales) provides a unique opportunity and a tough challenge to the high energy astrophysics community. In particular, the so-called DM indirect searches-mostly focused on a class of theoretically well-motivated DM candidates such as the weakly interacting massive particles-are affected by a complex astrophysical background of cosmic radiation. The understanding and modeling of such background require a deep comprehension of an intricate classical plasma physics problem, i.e., the interaction between high energy charged particles, accelerated in peculiar astrophysical environments, and magnetohydrodynamic turbulence in the interstellar medium of our galaxy. In this review we highlight several aspects of this exciting interplay between the most recent claims of DM annihilation/decay signatures from the sky and the galactic cosmic-ray research field. Our purpose is to further stimulate the debate about viable astrophysical explanations, discussing possible directions that would help breaking degeneracy patterns in the interpretation of current data. We eventually aim to emphasize how a deep knowledge on the physics of CR transport is therefore required to tackle the DM indirect search program at present and in the forthcoming years.
\end{abstract}

\section{Exotica: Where to Find Them?}

The particle dark matter (DM) [1] discovery may potentially undertake a different path than the beaten track of collider searches [2] and direct detection experiments [3]. Early Universe thermal relics, in particular, may be well-motivated DM candidates [4-6] expected to annihilate (or decay) even in today galactic halos, producing Standard Model (SM) particle yields. Therefore, the measurement of charged particle and gamma-ray fluxes of cosmic origin in a wide energy range - say from few $\mathrm{MeV}$ all the way up to the multi$\mathrm{TeV}$ domain - may be a quite unique tool at our disposal in order to probe the putative particle nature of DM [79].

It is widely recognized that the indirect extraction of a DM signal in this context is an extremely challenging task. The DM problem in general and the indirect searches in particular have been already presented in comprehensive review papers (see, e.g., $[10,11]$ ). Here, we wish to focus our attention on the important interplay between particle
DM signatures and the background signals expected from astrophysics, discussing in particular the phenomenological relevance of cosmic-ray physics.

As far as charged particles are concerned, the first consideration in order is that high energy protons, nuclei, and electrons are injected in copious amounts as cosmic rays (CRs) by different classes of astrophysical sources (such as shocks associated with supernova explosions or superbubbles or possibly accretion-powered mildly relativistic jets), which provide a huge and irreducible background. On the other hand, the paucity of antiparticles, mostly produced by secondary interactions of CRs, could in principle drastically improve the signal-to-noise ratio in favor of a putative DM detection, within a rather low expected astrophysical background; this possibility of detecting Early Universe relics by studying galactic antiparticles was first outlined in the early 1980s in several pioneering papers (e.g., $[12,13])$ and has been studied in much larger detail in particular during the last decade, mainly thanks to the dramatic improvement in the quality of the data provided by PAMELA [14] and 
AMS-02 [15] experiments. For a recent discussion on cosmic antimatter opportunities, see, for example, $[16,17]$.

Despite the low background, even in the case of antimatter searches the large uncertainties involved in the modeling of both conventional astrophysical production and galactic transport play a major role and have hindered a firm DM detection so far, although some recent tentative claims (in particular, among others, [18-22]) have triggered an important debate in the community. In Sections 4, 5, and 6 we will describe in detail the current status for positron, antiproton and antinuclei indirect searches: our purpose is to provide a case-by-case discussion mostly focused on the relevance of CR transport physics in antimatter channels for DM indirect detection.

While charged antiparticles may be promising indirect messengers of the particle DM nature, they do not retain the directionality from their emission point and, hence, cannot provide the morphological characterization of a DM signal: this possibility is accomplished instead with the analysis of gamma-ray data (for early studies, see, e.g., [23-25]; more recently, $[26,27])$. If DM particles eventually decay or annihilate into gamma radiation, it is crucial to identify the most promising regions of the sky where either the expected signal is large, or the astrophysical background is low: among the most important targets, we can certainly mention the inner galaxy (which satisfies the first criterion) and the DMdominated satellite galaxies orbiting around our galaxy. The most tentative claims and interesting bounds from the study of the gamma-ray sky will be reviewed in detail in Section 7.

Before going through an extensive discussion of all these channels for DM indirect searches, in the next two sections we will set up the stages of this review by briefly presenting some relevant aspects of DM models connected with the scope of the present paper and then highlighting the several key aspects of the galactic CR transport problem. Those concepts will be recalled all across the paper when the most relevant interpretations of CR and gamma-ray anomalies are discussed.

\section{Targeting DM Indirect Searches on High Energy Physics}

The quest for the fundamental origin of DM may require considering a priori an impressive range of energy scales (see $[28,29]$ for interesting historical retrospectives). For instance, sitting on the extremes of the viable mass window for DM searches, today we may be looking for imprints on the cosmological matter power spectrum of super-light candidates such as axion-like particles [30-32] from the string landscape $[33,34]$, as well as aiming to detect the gravitational echoes of massive black hole merging [35, 36], possibly originated from primordial density fluctuations in the Early Universe [37, 38].

However, the phenomenology of DM candidates may be intriguingly correlated to the long-standing puzzles pertaining to the realm of the SM (see, e.g., $[11,39]$ for broad reviews on the topic). Of particular significance, one of the main driving forces of research on High Energy Physics has been the quadratic UV sensitivity of the Higgs boson mass to any new physics energy scale above the electroweak one [40]. On general grounds, the attention of this review is mostly paid to DM candidates motivated by New Physics at the electroweak scale. Many extensions of the SM theory addressing the electroweak hierarchy problem can indeed accommodate such DM candidate in their spectrum; see, e.g., [5] for a review on the widely mentioned case of supersymmetry; [41, 42] for higher dimensional theories aimed at stabilizing the electroweak scale; [43-46] for examples of strongly coupled UV completions. Moreover, DM candidates sitting around the electroweak scale may also be well-motivated in the context of nonnatural theories addressing other possible $\mathrm{SM}$ issues, such as proton stability and/or gauge coupling unification; see, e.g., [47-49] for the case of supersymmetry; [50-53] for interesting universal/warped extra-dimensional proposals.

In all these scenarios, the DM particle is usually stable due to its charge under a (discrete) symmetry of the new theory, while a benchmark range of masses and couplings can be eventually individuated on the basis of the UV guiding principles. Most importantly, the emerging DM phenomenology from these studies typically falls in the experimental window of sensitivities for antimatter and gamma-ray searches discussed in the next sections.

Marginalizing over the specific details of UV models, the DM reference framework we mainly refer to, in this review, corresponds to Early Universe cold thermal relics. Assumed to be in thermal equilibrium with the primordial plasma at the very early stages, DM decoupling as nonrelativistic species eventually leads to [54] (for more details, see also [55]):

$$
\frac{\Omega}{0.25} \sim \frac{3 \times 10^{-26} \mathrm{~cm}^{3} \mathrm{~s}^{-1}}{\langle\sigma v\rangle} \simeq \frac{10^{-8} \mathrm{GeV}^{-2}}{\sigma} .
$$

$\Omega$ is the DM cosmological relative abundance observed today [56]; $\langle\sigma v\rangle$ is the DM thermally averaged particle annihilation cross section. In the last step we make use of the approximation $\langle\sigma v\rangle \simeq \sigma c / 3$, expected to be valid for a WIMP at the time of chemical decoupling in the Early Universe. By means of dimensional analysis, we can naively estimate the DM annihilation cross section in terms of its mass $m$ and dimensionless coupling constant $g$, obtaining

$$
m \sim\left(\frac{g^{2}}{10^{-1}}\right)\left(\frac{10^{-4} \mathrm{GeV}^{-1}}{\sqrt{\sigma}}\right) \mathrm{TeV}
$$

(1) in conjunction with the estimate in (2) characterizes the so-called Weakly Interacting Massive Particle (WIMP) miracle: a cold thermal relic charged under weak interactions and with mass close by the electroweak scale naturally accounts for the present DM abundance.

The WIMP mass range may be bracketed from below according to the seminal works in $[57,58]$ as $m \gtrsim 10$ $\mathrm{GeV}$, while it may be bracketed from the above unitarity arguments on the DM cross section pinpoint to $m \leqslant 10^{2} \mathrm{TeV}$ [59] (but caveats exist [60]). Typical expectations from the WIMP paradigm may be notably disregarded by the presence 
of resonant regimes, near mass thresholds, and coannihilation with other particles in the thermal bath [61]. Many other examples of variants to the standard WIMP freezeout scenario have been investigated in literature [62-70]. Nonperturbative effects such as Sommerfeld enhancement [71-74] and bound-state formation [75-80] have also more recently acknowledged to be of possible dramatic impact in the broad context of WIMP phenomenology.

Importantly, (1) shows that the DM relic abundance is mostly sensitive to the annihilation cross section. It follows that a WIMP-less miracle is perfectly conceivable [81], opening on general grounds a broader range of viable mass scales (for a supersymmetric setup, down to the $\mathrm{MeV}$ scale within gauge-mediation breaking) and couplings for the phenomenology of DM thermal relics. Therefore, indirect signals from WIMP-like scenarios-whose signatures have been comprehensively inspected in $[82,83]$ and are of particular importance for this review-are after all (1) intimately connected to the possibility of being effectively visible today and (2) possibly connected to the tantalizing discovery of New Physics near the electroweak scale [84].

\section{Miniguide to Galactic CR Physics}

3.1. Origin of CRs. A clear identification of the classes of astrophysical sources able to accelerate particles from $\mathrm{GeV}$ all the way up to $\mathrm{PeV}$ energies (and, for extragalactic accelerators, up to $\sim 10^{20} \mathrm{eV}$ ) is crucial for DM indirect detection.

We will not digress here on the long debate about the origin of CRs and their acceleration mechanisms (see, e.g., [85] for an excellent review). For the purpose of this review, let us start by mentioning the supernova paradigm as the main guideline. Supernova remnants (SNRs) were proposed as potential sources out of energy budget arguments in [86, 87]; the picture was better defined later in terms of SNRs located in our own galaxy $[88,89]$; however, a physical process capable of such a powerful CR acceleration had not been proposed yet at that time. Later, the theory of diffusive shock acceleration was presented in four famous seminal papers [90-93] and is currently considered the main reference framework in the field.

However, it is important to keep in mind that other classes of sources (e.g., pulsar wind nebulae for leptonic CRs [94], $\mathrm{OB}$ associations [95], and X-ray binaries [96]) and several other acceleration mechanisms have been proposed as well. As we will see below, many of these potential accelerators are expected to play a role in the explanation of some tentative claims of DM detection.

3.2. CR Transport: Preliminary Considerations. Let us now turn our attention to a crucial aspect of CR physics, which has an extremely relevant impact on DM indirect searches, i.e., CR propagation in our galaxy.

The usual starting point is a collection of several key observations that characterize the cosmic-ray flux.

(i) The isotropy of the arrival direction (at the level of $\sim 10^{-3}$ in the TeV-PeV range, recently measured with high precision by many experiments [97-102]). (ii) The much larger abundance of Lithium, Beryllium, and Boron compared to the solar system abundances, which is naturally interpreted as the signature of the interaction of primary species such as protons and heavy nuclei with a column density of interstellar gas as large as few $\mathrm{g} / \mathrm{cm}^{2}$ : such a grammage implies that the primary species have crossed the galactic disk many times.

(iii) The presence of a diffuse gamma-ray emission across the whole galactic disk, already predicted in the early 1960s [103] and first measured by pioneering satellite experiments such as OSO-3 (1967) and SAS-2 (1972); afterwards, COS-B and EGRET provided even more accurate results; nowadays, Fermi-LAT has provided a state-of-the art description of this emission in the 30 $\mathrm{MeV}-1000 \mathrm{GeV}$ range, as detailed below.

These pieces of information, combined together, suggest a "conventional scenario" for CR transport that was shaped by the pioneering work of Ginzburg and colleagues (see [104] and references therein and [105]), based on a random walk through the galaxy governed by the quasilinear theory of pitch-angle scattering on Alfvénic turbulence (QLT), first presented in the 1960s $[106,107]$.

3.3. The CR Transport Equation. Magnetohydrodynamic turbulence-which is ubiquitous in the interstellar medium (ISM) and covers a very wide range of scales from astronomical units (AUs) to kpc [108] - is widely considered as the main responsible for this diffusive regime. In more detail, the relativistic motion of charged particles in our galactic environment is affected by the presence of a coherent largescale magnetic field component, $\vec{B}[109,110]$, on top of which magnetic inhomogeneities, $\overrightarrow{\delta B}$, are propagating. These fluctuations in the magnetized interstellar medium (ISM) are associated with a turbulent cascade that is believed to be either initiated at large scales $\sim 10^{2} \mathrm{pc}$ (by supernova explosions, differential rotation of the galactic disk, or other mechanisms [111]) or (especially at small scales) triggered by CR themselves via streaming instability [112, 113]. This cascade has been usually considered, in the basic scenario of QLT, as isotropic and mainly composed of Alfvén waves, i.e., transverse magnetic perturbations moving at the Alfvén speed $^{1}$ :

$$
v_{A} \simeq 2 \times 10^{6} \frac{|\vec{B}|}{\mu \mathrm{G}} \sqrt{\frac{\mathrm{cm}^{-3}}{\rho_{\text {ISM }}}} \mathrm{cm} \mathrm{s}^{-1} .
$$

Following in part the approach of [114], we recap here the main features of QLT (see also [104, 105]).

The rationale of QLT is to consider the interaction of a charged particle of momentum $\vec{p}=m \vec{v}$ with magnetic inhomogeneities $\delta \vec{B}$ that are sufficiently small (with respect to the regular field $\vec{B}$ ) at the scale of interest. The process is well described by a stochastic equation for the pitch angle, defined as $\mu=\cos (\widehat{p} \wedge \vec{B})$. On average the variance of the pitch angle can be shown to feature a resonance condition 
[105]. According to it, the particle only interacts with the inhomogeneities of wavelength $\sim 2 \pi / k$ matching the particle Larmor gyroradius $r_{L}{ }^{2}$ :

$$
\left\langle\frac{\Delta \mu \Delta \mu}{\Delta t}\right\rangle=\frac{\pi v}{\mu r_{L}^{2}} \frac{|\overrightarrow{\delta B}|^{2}}{|\vec{B}|^{2}}\left(1-\mu^{2}\right) \delta\left(k-\frac{1}{\mu r_{L}}\right) .
$$

Let us now consider an ensemble of particles described by a phase-space distribution $f(\vec{x}, \vec{p}, t)$, with probability density $\Psi(\vec{p}, \Delta \vec{p})$ for transitions $\vec{p} \longrightarrow \vec{p}+\Delta \vec{p}$ in momentum space, due to interactions with stochastic fluctuations in the magnetized environment. We can state that, after a lapse $\Delta t$, in the Alfvén wave rest frame (primed), the evolved phasespace distribution must be equal to

$$
\begin{aligned}
f & \left(\vec{x}^{\prime}+\vec{v}^{\prime} \cdot \Delta t, \vec{p}^{\prime}, t+\Delta t\right) \\
& =\int d \Delta \vec{p}^{\prime} \Psi\left(\vec{p}^{\prime}-\Delta \vec{p}^{\prime}, \Delta \vec{p}^{\prime}\right) f\left(\vec{x}^{\prime}, \vec{p}^{\prime}-\overrightarrow{\Delta p}^{\prime}, t\right)
\end{aligned}
$$

with $\vec{v}^{\prime}$ being the CR particle velocity in the wave frame.

We assume that detailed balance holds; i.e., a transition $\vec{p}^{\prime} \longrightarrow \vec{p}^{\prime}-\Delta \vec{p}^{\prime}$ described by a probability $\Psi\left(\vec{p}^{\prime},-\overrightarrow{\Delta p}^{\prime}\right)$ is equivalent to the one described by $\Psi\left(\vec{p}^{\prime}-\Delta \vec{p}^{\prime}, \Delta \vec{p}^{\prime}\right)$. Applying this principle in the limit of $\Delta p^{\prime} / p^{\prime} \ll 1$ (originating from $|\overrightarrow{\delta B}| /|\vec{B}| \ll 1)$ that characterizes QLT, we can write

$$
\left\langle\Delta p_{i}^{\prime}\right\rangle_{\Delta \vec{p}^{\prime}}=\frac{1}{2} \sum_{j} \frac{\partial}{\partial p_{j}^{\prime}}\left\langle\Delta p_{i}^{\prime} \Delta p_{j}^{\prime}\right\rangle_{\Delta \vec{p}^{\prime}},
$$

where $\langle\ldots\rangle_{\Delta \vec{p}^{\prime}} \equiv \int d \Delta \vec{p}^{\prime} \Psi\left(\vec{p}^{\prime}, \Delta \vec{p}^{\prime}\right)$ and a Taylor expansion of $\Psi\left(\vec{p}^{\prime}, \Delta \vec{p}^{\prime}\right)$ has been performed.

Assuming the static limit, i.e., $\Delta t / t \ll 1$, starting from a Taylor expansion of (5), with the help of (6) we finally get, in the same wave frame,

$$
\frac{\partial f}{\partial t}+\vec{v}^{\prime} \frac{\partial f}{\partial \vec{x}^{\prime}}=\frac{\partial}{\partial \vec{p}^{\prime}}\left(D_{\vec{p}^{\prime} \vec{p}^{\prime}} \frac{\partial f}{\partial \vec{p}^{\prime}}\right) .
$$

This is a Boltzmann equation where in the right-hand side the "collision operator" qualifies Brownian motion in momentum space with diffusion coefficient

$$
D_{p_{i}^{\prime} p_{j}^{\prime}} \equiv \frac{1}{2}\left\langle\frac{\Delta p_{i}^{\prime} \Delta p_{j}^{\prime}}{\Delta t}\right\rangle_{\Delta \vec{p}^{\prime}} \text { for } i, j=1,2,3,
$$

describing indeed the momentum isotropization due to CR stochastic scattering with Alfvén waves. ${ }^{3}$

Let us now perform a transformation to the galactic rest frame. In this frame of reference, (7) features a spatial diffusion operator along the direction of the regular field as well. This is the most important term that governs CR transport in the galaxy. The spatial diffusion coefficient $D_{z z}$ is related to $D_{p p}$ by [115]

$$
D_{z z} D_{p p} \propto v_{A}^{2} p^{2},
$$

for $\vec{B}=B \widehat{z}$. Inspired and guided by the results of QLT, a general transport equation is usually considered, mainly based on the aforementioned process of diffusion in both position and momentum space, but featuring a wider set of terms associated with other physical phenomena. The full equation reads

$$
\begin{aligned}
\frac{\partial N}{\partial t}+ & \vec{\nabla}(\vec{u} N)-\frac{1}{3} \frac{\partial}{\partial p}[p(\vec{\nabla} \cdot \vec{u}) N] \\
& -\vec{\nabla}\left(D_{\vec{x} \vec{x}} \vec{\nabla} N\right)-\frac{\partial}{\partial p}\left[p^{2} D_{p p} \frac{\partial}{\partial p}\left(\frac{N}{p^{2}}\right)\right] \\
& +\frac{\partial}{\partial p}\left(\frac{d p}{d t} N\right)=Q_{0}+Q_{\text {sec }}-\frac{N}{\tau_{N}},
\end{aligned}
$$

with $N(\vec{x}, p, t)$ and $Q(\vec{x}, p, t)$ being, respectively, the CR density species and CR injecting density source per unit of momentum. In the left-hand side, the diffusion term is usually isotropic and described by a scalar, positionindependent coefficient, despite the fact that QLT predicts a highly anisotropic transport along the regular field direction (see, e.g., the discussion in [116]). The scalar spatial diffusion coefficient is generally taken as

$$
D=\frac{c r_{L}}{\mathscr{F}(k)}
$$

where $\mathscr{F}(k)$ is defined as the (normalized) power associated with the turbulent modes with wave number $k \propto 1 / p$ resonating with the particles carrying momentum $p$. Since the turbulent power scales as a power law, the rigidity dependence of the diffusion coefficient is usually parametrized as

$$
D=D_{0}\left(\frac{p}{p_{0}}\right)^{\delta}
$$

with $D_{0}$ and $\delta$ as free parameters to be fixed by comparison with CR data. The spatial dependence of such normalization that stems from the spatial variations of the turbulent power is usually neglected with some relevant exceptions [116-121].

The momentum diffusion is also called stochastic reacceleration, and the relation 10 is assumed to hold.

The left-hand side also involves an advection term originally present in (7) as well, now characterized by the bulk velocity of the plasma in the lab frame: galactic winds affecting CR motion may be described by such a term, together with adiabatic energy losses, involving velocity gradients even of $O\left(10^{2}\right) \mathrm{km} \mathrm{s}^{-1} \mathrm{kpc}^{-1}$ perpendicularly to the galactic disk.

The physics of advective-diffusive transport is enriched by two more relevant phenomena included in (10): net energy losses and spallation. In fact, we need to consider CR energy loss processes, characterized by the continuous loss rate $d p / d t$, particularly important, e.g., at high energies for light charged species such as leptons (see, e.g., $[122,123])$.

Eventually, on the right-hand side of (10),

(i) the primary source term $Q_{0}$ captures the primary accelerators of CRs: as mentioned above, while the supernova paradigm is still the most accredited one, other classes of sources can certainly be at work; 
(ii) the secondary source term $Q_{\mathrm{sec}}$ describes the production of a given species from spallation of the heavier ones onto interstellar gas;

(iii) a loss term due to inelastic collisions characterized by an interaction time $\tau_{N}$ is also introduced.

An important remark is needed at this point. Our picture of MHD turbulence has dramatically improved during the latest decades: according to the current scenarios [124, 125], MHD turbulence is composed of an anisotropic cascade of both Alfvén waves, and isotropic fast magnetosonic modes, as theoretically demonstrated and numerically confirmed by several simulations. As a consequence of the anisotropy of the Alfvénic cascade, the scattering efficiency on Alfvén waves turns out to be very low [126], posing a tough challenge to the whole scenario discussed above. Among others, a possible solution $[127,128]$ is that magnetosonic modes dominate gyroresonance interaction for most of the pitch-angle range.

However, although the actual microphysics underlying the CR random walk is still far from being exhaustively addressed, the QLT can still be considered a useful guideline to be taken as a reference, and (10) should be understood as a phenomenological tool to tame the complexity of the plasma physics problem, allowing us to make predictions against a plethora of data.

3.4. Modeling CR Transport: A Glimpse. In order to solve the complicated CR transport equation (for each CR species), today we have at our disposal several public numerical codes, equipped with different numerical and astrophysical ingredients, aimed at solving (10), most notably (in chronological order): GALPROP[129-132], DRAGON [123, 133-135], and PICARD $[136,137]$. A semianalytical approach is instead followed by the USINE project [138].

While a detailed and realistic study of galactic CR propagation requires the extensive use of those numerical or complex semianalytical methods, we can extract some physical insight useful for the next sections looking at a simplified version of (10).

Indeed, at the basis of CR transport, an important hierarchic game of scales may be conceived: convective and reacceleration effects are typically related to low-energy regimes, while energy loss rates are negligible for high energy hadrons such as protons or heavier nuclei. So, for energies $E \gtrsim$ few $\mathrm{GeV}$, the galactic motion of heavy species can be approximately described to be in a purely diffusive regime: then, we may trade the spatial diffusion operator for an effective time of confinement, $\tau_{D}$, i.e.,

$$
\vec{\nabla}\left(D_{\vec{x} \vec{x}} \vec{\nabla} N\right) \longrightarrow \frac{N}{\tau_{D}}
$$

and treat the galaxy as a box where CRs perform a random walk-up to the box boundaries, beyond which they leak out. In the steady-state limit of this simplified framework, CR secondary species, produced by the interaction of sourceinjected (namely, primary) CR particles, can be estimated as

$$
N_{s}=Q_{s} \tau_{D} \propto N_{p} \tau_{D}
$$

Hence, the timescale for confinement of CRs in the galaxy is intimately linked to relative abundances of secondary and primary species. As anticipated in the above, antimatter species are typically produced as secondaries, and therefore (14) is of direct relevance for DM indirect searches in antimatter channels.

Note that, by dimensional analysis, $\tau_{D} \sim H^{2} / D$, where $H$ captures the typical size of the box. Therefore, secondary fluxes are sensitive to both the spatial diffusion coefficient and the height of the CR propagation halo. As we will discuss below, our current poor knowledge of the value of $H$ generally translates into an important source of uncertainty in DM indirect detection studies.

As stated above, the spatial diffusion coefficient scales as a power law with rigidity. Supplementing (14) with such expectation, we have a theoretical prediction that nicely fits the trend of available experimental data [140]: at energies above few $\mathrm{GeV}$, the measurements of local CR secondaryover-primary observables like $\mathrm{B} / \mathrm{C}$ are indeed compatible with a power-law behavior (with index $\delta$ ) of the diffusion coefficient. Typical estimates of this parameter are in the range $0.3<\delta<0.6$ [141-145], with a normalization at $\mathrm{GeV}$ corresponding to $D_{0} \simeq 10^{28} \mathrm{~cm}^{2} \mathrm{~s}^{-1}$, but pertain only to a local measurement (see [118] for a possible indirect inference of $\delta$ across the galaxy).

\section{The Antiproton Channel}

In the conventional scenario sketched above, antiprotons are produced in the galactic environment by spallation of heavy nuclei and protons onto interstellar gas.

The early measurements of the antiproton flux date back to the 1970s and early 1980s; a first tentative claim of anomaly with respect to the conventional expectations based on the picture of nuclear spallation goes back to [146]. However, DM connections were not outlined at that time: cosmic antiprotons were considered a promising channel for DM searches only some years later, in several seminal papers (see, for instance, $[12,147,148]$ and, more recently, [149]).

A dramatic improvement in the accuracy of the data was provided by the PAMELA collaboration in 2009 [150] (with further refinement in [151]): the measurement showed a reasonably good agreement with conventional models based on purely secondary origin, as confirmed by [141, 152]. A note here is in order about the meaning of conventional model: in what follows, we will use this expression for a model based on the simplest version of (10), taking a single class of sources (SNRs) at work, with antiparticles produced only as secondary products from primary spallation, and featuring constant and homogeneous diffusion, tuned on local CR data.

Given the absence of significant unexpected spectral features (such as bumps), the potential constraining power of PAMELA dataset for DM searches was soon demonstrated in a series of papers (from the early ones as $[153,154]$ to the more recent [155]), which provided a comprehensive discussion on the upper bound on the WIMP annihilation cross section from the detection of cosmic antiprotons. The most relevant point made in those papers is the crucial role of CR transport. 
A major source of uncertainty, in particular, is the size of the diffusive halo $H$ introduced in the previous section, i.e., the volume, where galactic CRs are effectively confined by the presence of a turbulent magnetic field. Models of CR transport based on larger diffusion halos usually feature larger average values of the diffusion coefficient in order to correctly reproduce the secondary/primary ratio data: therefore, in these scenarios, the antiproton flux probes a larger region of the galaxy and exhibits a larger constraining power. On the other hand, cases where one assumes a very thin halo (smaller than $\simeq 2 \mathrm{kpc}$ ) turn out to be much less restricting on the particle DM properties indirectly probed.

Another source of uncertainty certainly lies on the properties of CR transport in a much smaller environment: the Heliosphere. We refer to [155] and references therein for a comprehensive discussion on this aspect.

As a consequence of this complicated puzzle, it was not possible to firmly exclude some relevant tentative DM claims made in other channels (see the gamma-ray section for more details), and the most severe limitation came from the poor constraints we actually have on the size of the galactic CR diffusion halo. A possible improvement in this direction may come from more accurate measurements of the Beryllium isotopes and from a careful analysis of the current and forthcoming data on the vertical profile (with respect to the galactic plane) of the synchrotron emission from the galaxy (following $[156,157]$, we point out that the current data seem to favor large values for the size of the diffusion halo).

In 2015 a much more accurate dataset was published by the AMS-02 collaboration [20]. The debate on the antiproton channel has then included tentative claims of anomalies with respect to the conventional scenario, possibly explained in terms of DM indirect detection.

First of all, the AMS-02 collaboration itself initially claimed the presence of an excess at high energies over $100 \mathrm{GeV}$. Right after, the significance of this anomaly was better characterized $[139,143,158]$, pointing only to a mild overshooting of the expected background. The relevance of this putative discrepancy and the estimated impact of the different sources of uncertainty on the model predictions is well depicted in Figure 1 (taken from [139]). ${ }^{4}$

Taking at face-value the original tentative claim from AMS, the interpretation of the excess requires scenarios beyond the conventional on of $\mathrm{CR}$ transport pictured in the miniguide of Section 3. For instance, a mechanism that may be at work and explain the discrepancy is the secondary production at the accelerator: the idea, proposed before AMS data in [159] as a possible explanation to the positron ratio anomaly (see Section 6), is that secondary products of hadronic interactions inside the sources can participate in the acceleration process and subsequently escape into the interstellar medium as an extra component featuring a very flat spectrum. In [160] this scenario was applied to (pre-AMS) antiproton data as well, and the authors demonstrated that the boron-over-carbon ratio has much constraining power for this interpretation.

More recently, refined Bayesian analyses that include the official AMS data on this observable as well (see, e.g., [144]) confirm the presence of a mild discrepancy between the

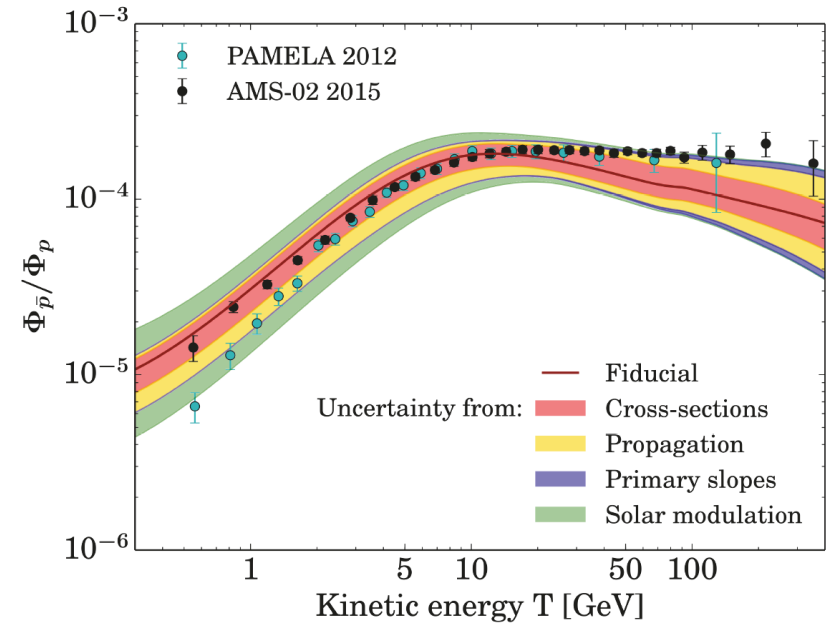

FIGURE 1: Impact of cross section, CR transport, and modulation uncertainties on the conventional predictions for the antiproton compared to the related dataset collected by the AMS- 02 collaboration. Figure 1 is reproduced from Giesen et al. (2015) [139] (under the Creative Commons Attribution License/public domain).

regions of the parameter space pointed by AMS antiproton and $\mathrm{B} / \mathrm{C}$ data.

However, spatial-dependent diffusion setups (e.g., the phenomenological two-zone models as those considered in $[161,162]$, designed to capture both CR transport in preexisting SNR-driven galactic turbulence, and confinement by CR-driven turbulence via streaming instability) seem to solve the discrepancy, as well as the latest scenarios that include secondary production at the accelerator [163].

DM interpretations for the high energy discrepancy are also still viable for quite large values of the DM particle mass (as shown, e.g., in [164-166]), in particular for light mediator scenarios. At the same time, the constraining power of AMS data in the energy range where no relevant feature or anomaly is present has been most recently explored in $[167,168]$.

On the other hand, on the low-energy side, a possible indication of a DM signal for DM masses near $80 \mathrm{GeV}$ has been found [168-170], with a hadronic annihilation cross section close to the thermal value: interestingly, this tentative claim is compatible with the DM interpretation of the galactic center gamma-ray excess (see Section 7). Again, more investigation of the transport uncertainties (both in standard and beyond-standard scenarios) and more detailed combined studies of this signal together with constraints from other probes (e.g., the observation of dwarf spheroidals in the gamma-ray band) will be crucial in order to confirm the existence of this anomaly.

\section{The Avenue for Antinuclei}

A milestone campaign for imprints of particle DM on the observable CR radiation may correspond to the discovery of galactic light antinuclei such as antideuteron $\left({ }^{2} \overline{\mathrm{H}}\right)$ [171-174] and antihelium-3 $\left({ }^{3} \overline{\mathrm{He}}\right)[175,176]$. At present, no compelling evidence for a detection of antimatter with mass number 
$A \geq 2$ has been experimentally corroborated in the measurement of galactic CR fluxes. A notable upper limit on the flux of cosmic antideuteron has been set by the BESS facility-reporting at the $95 \%$ of confidence level $\Phi_{2 \overline{\mathrm{H}}} \lesssim 2 \times$ $10^{-4}\left(\mathrm{~m}^{2} \mathrm{~s} \mathrm{sr} \mathrm{GeV} / \mathrm{n}\right)^{-1}$ for kinetic energy per nucleon $0.17 \leq$ $\mathrm{T} \leq 1.15 \mathrm{GeV} / \mathrm{n}$ [177] - while the BESS-Polar collaboration currently constrains antihelium-to-helium flux to be smaller than $10^{-7}$ in the interval of probed rigidities, $1.6-14 \mathrm{GV}$ [178].

Today, the AMS-02 mission is operating in the direction to perform the first historical observation of cosmic light antinuclei, with promising projected sensitivities [179]. A tentative claim of few antihelium events-possibly measured by the AMS- 02 collaboration-might be already at hand, waiting for a firmer experimental response in the upcoming years; see, for instance, [180].

The phenomenological relevance of the avenue for antinuclei in relation to DM indirect searches stems from the kinematics of spallation processes producing CR secondaries: the energy threshold associated with the production of one antideuteron is roughly 2.5 greater than the one required to produce a secondary antiproton. Moreover, the same energy threshold is a monotonic increasing function of the mass number $A$. Therefore, the low-energy flux of cosmic antinuclei is expected to be small [181], opening a low-energy window-related to kinetic energies per nucleon between $\sim 0.1$ and few $\mathrm{GeV} / \mathrm{n}$-for large signal-to-background ratios from exotica.

The physics of CR accelerators and of propagation impacting the prediction for antinuclei fluxes at Earth sits on the very similar grounds of the one discussed for the antiproton channel. On the one hand, the unknowns stemming from galactic CR propagation affecting the predicted galactic fluxes for antideuteron and antihelium should be correlated to the galactic antiproton spectrum and boron-to-carbon ratio data. On the other one, the injection of antinuclei in the galactic interstellar medium is intimately connected to the antiproton production mechanism. As discussed in the previous section, the latter may be sourced from in situ acceleration in the downstream region of supernova remnant shockwaves. Such mechanism generally yields a source term distinguished into a A-component, referred to as CR secondaries accelerated by the shock, and a B-component, related to standard spallation processes sourced by CR primaries. Most recent analyses on the topic have included also these extra contributions in their predictions [182-184].

Figure 2, from [182], reports the maximum contribution to the antideuteron flux with respect to the typical unknowns from CR physics, properly calibrated on antiproton AMS-02 data. The components from production in supernovae (SNRA and SNR-B bands) are found to be subdominant, giving (at most) a $10 \%$ effect with respect to the main standard component, obtained by considering the interactions of CR primaries with the interstellar medium (black line). Figure 2 also shows predictions for some benchmark exotica such as annihilating DM particles with masses of $\mathcal{O}(100) \mathrm{GeV}$ and $100 \%$ branching ratios into $b \bar{b}$ or $W^{+} W^{-}$final states, together with the expectations from exotica that gained recent interest $[35,36]$ such as primordial black holes $[185,186]$. The plot

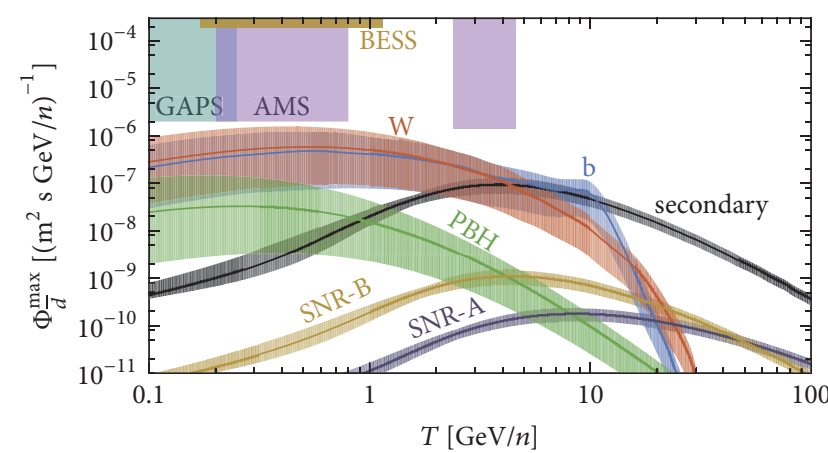

Figure 2: Maximum ${ }^{2} \overline{\mathrm{H}}$ flux compatible with AMS-02 data from evaporation of primordial black holes (green) and DM particle annihilation (blue and brown), compared with current and projected sensitivities from BESS, AMS, and GAPS experiments. Maximum flux for the expected astrophysical background is also shown: in black color the contribution from CR spallation processes with the interstellar medium and the subleading SNR-A and SNR-B ones. Bands bracket uncertainties from force-field approximated solar modulation and an event-by-event coalescence model. Figure 2 is reproduced from Herms et al. (2016) [182] (under the Creative Commons Attribution License/public domain).

clearly highlights the importance in the aforementioned low-energy window. However, contrary to common wisdom [179], Figure 2 also underlines the unlucky possibility that within the forthcoming years current and future experimental facilities may not be sensitive to antideuteron yields from $\mathrm{DM} /$ exotica production.

At this point, it is important to stress that unknowns stemming from the physics of CR accelerators and galactic transport should not be retained to be the major source of uncertainty in the prediction of cosmic antinuclei fluxes. The bands reported in Figure 2, while including the effects of solar modulation-typically studied within the force-field approximation [188] and better investigated in [189] by means of numerical tools [190, 191] —are most importantly related to the coalescence model adopted to establish antideuteron formation. At present, the prediction of antinuclei fluxes seems to strongly depend on the assumptions made to describe antiproton-antineutron fusion [179]. Nowadays, state-of-the-art analyses can avoid to rely on simplistic analytical modeling [192], making instead use of Monte Carlo event generators and available data from colliders. Large systematics on the formation of antideuteron and antihelium can be understood on the basis of the sensitivity of these studies on the hadronization model implemented and the experimental dataset considered; see, e.g., $[179,193]$. Interestingly, a recent work focused on the description of nuclear coalescence via a physical modeling for the fusion of nucleons into composite nuclei [194], and exploiting twoparticle correlation measurements [195] has pointed out the possibility that the production cross section in pp collisions for antihelium-3 may have been underestimated by up to two orders of magnitude. Consequently, a putative detection of cosmic ${ }^{3} \overline{\mathrm{He}}$ events related to kinetic energies greater than 1 $\mathrm{GeV} / \mathrm{n}$ may actually be within the reach of AMS-02 in few years [17]. 
Therefore, we may conclude that searches for antinuclei, while being potentially exposed to CR propagation details, are mainly plagued by the assumptions and the systematics involved in the estimate of the poorly known production cross section. This can have a dramatic impact on our present ability to make projections for a concrete signal detection of antideuterons and antihelium-3. At the same time, these uncertainties leave us hope to foresee a spectacular discovery of exotica such as DM in the peculiar window of low-energy antinuclei events.

\section{The Positron Channel}

The positron channel has been under the spotlight for a long time in the DM indirect detection community. As mentioned in Section 1, the paucity of those particles makes them an ideal target for DM searches, and the presence of a significant anomaly (with respect to the conventional expectations) has further increased the interest around this observable during the latest decade.

Let us start by clarifying, also in this case, what we mean by "conventional" predictions. In the context of a simplified treatment of acceleration and transport-as described in the miniguide of CR physics-a slightly different discussion is actually required for leptons in general. In fact, high energy leptons feature relevant energy losses especially at high energies, which implies a new timescale competing with the diffusion one. The simplified transport equation governing $e^{ \pm}$ propagation is therefore of this kind:

$$
\frac{\partial N}{\partial t}-D \Delta N-\frac{\partial}{\partial p}\left(\frac{d p}{d t} N\right)=Q
$$

where inverse Compton scattering on the photon background and synchrotron radiation define the typical timescale for energy losses, $\tau_{E_{\text {loss }}}(p) \sim p /|d p / d t| \propto p^{-1}$. The solution of (15) is given by a Green function that boils down to

$$
N \simeq \frac{Q(p) \tau_{E_{l o s s}}}{\sqrt{D(p) \tau_{E_{l o s s}}}}
$$

For primary electrons this result implies a scaling $\propto$ $\mathrm{p}^{-\alpha_{e}-\delta / 2-1 / 2}$, where $\alpha_{\mathrm{CR}}$ is the CR injected spectral index.

In the standard scenarios, positrons in the galaxy are believed to originate, like antiprotons, as an entirely secondary component arising from the collisions of relativistic protons with the ISM gas according to a chain like $p+H \longrightarrow \cdots \longrightarrow$ $\pi^{ \pm} \longrightarrow \mu^{ \pm}+\cdots \longrightarrow e^{ \pm}+\ldots$. The source function of positrons is then expected to scale as

$$
q_{e^{+}} \propto N_{p} n_{H} \sigma_{p \longrightarrow e^{+}} \propto Q_{p} \tau_{\text {diff }} \propto p^{-\alpha_{p}-\delta}
$$

where we have implemented the rather simplistic approximation of an energy independent cross section $\sigma_{p \longrightarrow e^{+}}$, used the fact that the relevant timescale for propagation of high energy protons is the diffusion timescale, and defined $\alpha_{p}$ as the spectral index for the proton injection source function.
Plugging this result in (17), we can predict the scaling of the propagated positron flux over the electron one:

$$
\frac{N_{e^{+}}}{N_{e^{-}}} \propto p^{-\alpha_{p}+\alpha_{e}-\delta},
$$

with $\alpha_{e}$ being the spectral index at injection for the electron source distribution.

In the framework of diffusive shock acceleration, the injected spectral index should not differ much among different species. Consequently, the ratio of secondary positrons over primary electrons is predicted to decrease with increasing energy, unless a (very unlikely) large difference between the source spectral indexes for protons and electrons is assumed ad hoc.

The rise at high energy in the positron fraction originally discovered by PAMELA in 2009 [18] and subsequently confirmed by Fermi-LAT and AMS-02 [19] collaborations constitutes then a substantial deviation from the standard prediction of (18) and appears robust with respect to uncertainties in CR transport models, implemented in a more realistic way (see, however, [196]). The release of the data on the absolute positron spectrum [197] confirmed and strengthened this conclusion.

The detection immediately triggered a debate in the community (see, e.g., $[198,199]$ and references therein). A natural explanation in terms of nearby astrophysical accelerators of primary $e^{+}+e^{-}$pairs, e.g., pulsar wind nebulae (already invoked in [94] as potential contributors to the leptonic flux), was soon considered as a very promising one; see Figure 3 for a recent realization of this scenario, compared to up-to-date experimental data. Other astrophysical interpretations were proposed (see, e.g., [200]), including the already mentioned secondary production at accelerators [159].

On the other hand, many DM interpretations were invoked as well: the tough challenges for model building are in this case the large annihilation cross section required to sustain the measured positron flux at high energy and the strong constraints originating from other channels (including gamma rays, CMB, and antiprotons); we refer [201] for an early review on the topic. The Early Universe bounds $[202,203]$ are particularly difficult to evade, and one has to invoke, for instance, nonthermal mechanisms (see, e.g., [204] for a recent two-component scenario in which the heavier DM species is produced as a thermal relic in the Early Universe and decays to the lighter species over cosmological timescales) or Breit-Wigner enhancement of present-time annihilation (already proposed in 2009 to explain the boost factor required by PAMELA data $[205,206]$ and recently reconsidered, e.g., in [207, 208], as a way to evade the stringent $\mathrm{CMB}$ constraints).

More interestingly, nowadays it is possible to challenge the widely debated pulsar hypothesis in several ways, and the uncertainties in CR transport play a major role.

First of all, it is possible to look for an anisotropy in the arrival direction of high energy leptons. The FermiLAT collaboration has released two papers in 2010 and 2017 on this topic, with stringent upper limits [209, 210]. The compatibility between the pulsar models and those limits has 

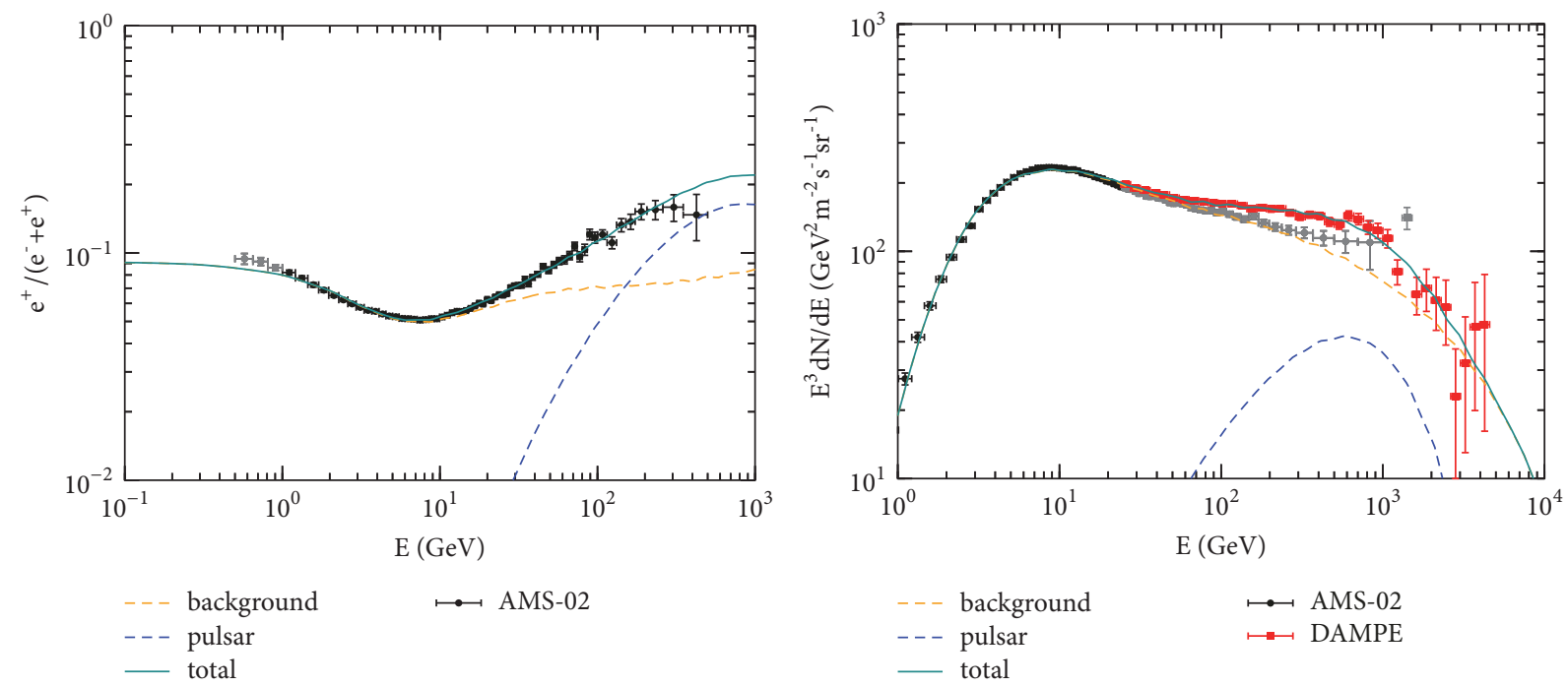

Figure 3: The most recent leptonic data from AMS and DAMPE, interpreted within a pulsar scenario. Figure 3 is reproduced from Yuan et al. (2017) [187] (under the Creative Commons Attribution License/public domain).

been studied several times in the literature (see, e.g., [211]) and is still matter of debate: as shown recently in [212], it is possible to build scenarios that are compatible with the most recent upper limits, where few nearby pulsars contribute at high energy and a collection of more distant ones dominates the low-energy positron flux; see also [213]. We also stress that it is problematic to rule out any pulsar scenario exploiting the anisotropy upper limits alone, due to the fact that we can only observe a subset of the nearby pulsar in radio and gamma rays, given the highly collimated emission (see [214]).

Moreover, the gamma-ray observatories may now allow to identify the emission from the leptons leaving nearby known pulsars. Along this track, a detection of a TeV halo around Geminga has recently been reported in [215]: in that paper a naive estimate of the diffusion coefficient in the vicinity of Geminga is presented, which turns out to be much smaller than the average galactic one inferred by secondary-to-primary ratios, posing a challenge both to CR transport models and to the pulsar interpretation of the positron anomaly as well; see also the follow-up detailed discussion in [216].

Very recently, the antiproton and positron channels were critically reexamined in [217]. In that paper it is noticed that the ratio between the positron (or antiproton) flux to the proton one is consistent with the secondary production rates in the conventional picture. Based on these considerations, the author suggests that galactic positrons and antiprotons may have a common origin as secondaries in hadronic interactions, probably produced in the local interstellar environment, so that diffusion and energy loss do not act for enough time to leave an observable imprint on the spectrum. If confirmed, this would imply a completely different propagation scenario characterized by a much lower residence time $(\sim 1 \mathrm{Myr})$ compared to current benchmark values: such scenario would also accommodate the spectral break in the electron spectrum reported by HESS [218], but not the electron slope, which is actually steeper than the antiproton and positron one: in such alternative framework, the $e^{-} / p$ discrepancy could in principle be generated at source, by not-yet identified mechanisms. The take-home message of this discussion is that the positron channel is far from being understood, and the nature of the emission above $\simeq 30 \mathrm{GeV}$ remains mysterious. However, DM interpretations of this anomaly seem disfavored with respect to several alternative astrophysical scenarios, in particular the pulsar hypothesis. Possible avenues towards a clearer understanding of these issues are (1) more detailed studies of the leptonic CR anisotropy (that can in principle provide a smoking gun of the pulsar scenario or in alternative strongly constrain the scenario itself); (2) more data beyond $\mathrm{TeV}$ energies: experiments such as DAMPE ${ }^{5}$ and CALET ${ }^{6}$ are already operating, and the first results from DAMPE [219] already showed some interesting features to be confirmed and further investigated [187] (as shown in Figure 3), while the measurement on $e^{+}+e^{-}$ spectrum from $10 \mathrm{GeV}$ to $3 \mathrm{TeV}$ has been recently released by CALET collaboration [220]; (3) more investigations of the interplay with the high energy gamma-ray observations, such as the $\mathrm{TeV}$ halo around Geminga, aimed at characterizing the diffusion properties of leptons in the vicinity of the sources; (4) also in this case, a better characterization of the diffusion halo size, for instance, by means of analyses focused on the radio emission, following, e.g., the approach of $[156,157]$.

\section{Gamma-Ray Opportunities}

The ubiquitous flux of high energy CR nuclei and leptons may be able to transform the galaxy into a huge pion factory and an efficient machine to up-scatter diffuse photons emitted by stars and reprocessed by dust grains. These processes, namely, $\pi^{0}$ decay and inverse Compton scattering, with the addition of the (usually subdominant) bremsstrahlung emission, yield a diffuse flux of high energy photons from the $\mathrm{MeV}$ to the 
multi-TeV energy domain, reaching the current sensitivity of space missions such as Fermi-LAT and AGILE, and ground-based facilities such as HESS, MAGIC, VERITAS, and HAWC.

Therefore, the observable gamma-ray sky can give us today a quite unique diagnostics of $\mathrm{CR}$ transport far from the solar system environment. The galactic diffuse gamma-ray emission stemming from CR interactions with the ambient gas and radiation field constitutes indeed the bulk of photons measured along the galactic plane region [221]. It depends on our observational knowledge of emitting targets [222], namely, the indirect tracing of gas column densities [223, 224] through, e.g., observed CO emissivities, or the characterization of the low-energy photon background, the socalled interstellar radiation field [225]. Moreover, it crucially relies on the details about CR propagation across the galaxy [226].

In the last few years important progress has been pursued in the development of phenomenological viable models for galactic CR propagation, able to match the observed $\mathrm{GeV}-\mathrm{TeV}$ photon data from the galactic plane region, while reproducing local $\mathrm{CR}$ measurements $[117,118]$. In the next future, a more systematic study of gamma-ray data in symbiosis with the analysis of local CR observables may offer us the most important chance to pin down the exact features underlying (10) (see, for example, $[116,227]$ ) in a data-driven fashion [162, 228-230].

Looping over uncertainties both on the side of emitting targets and possibly also on the underlying CR transport properties is a challenging task $[221,231]$. However, such an attempt is particularly welcome in order to constrain particle DM properties [226, 232, 233]. As previously mentioned, anomalies in the gamma-ray sky may be extremely compelling for indirect DM searches. State-of-the-art N-body simulations (see, e.g., [234, 235]) predict an extended DM halo embedding and surrounding the Milky Way, with a central density peaking in correspondence to the galactic center (GC). Then, within a scenario where DM particles pair-annihilate (or decay) eventually to gamma-ray photon yields, the GC region is likely the brightest possible target for DM indirect searches, being relatively close to us $(\sim 8$ $\mathrm{kpc})$.

The kinematics of DM thermal relics annihilating today in the halo may actually give rise to peculiar photon energy spectra. In particular, gamma-ray photon lines would not have any well-known astrophysical counterpart. Therefore, monochromatic lines in the $\mathrm{GeV}-\mathrm{TeV}$ range produced by $\mathrm{DM}$ particle pairs annihilating into two-body final state channels with one or two photons give rise to a potential smokinggun signature. However, in a standard scenario of electrically neutral particles [236], DM rate to monoenergetic photons will exhibit loop-suppression (see, e.g., [237, 238] for the benchmark of neutralino DM). Interestingly, a hint in favor of such a spectacular signature has been found in 2012 from dedicated analyses of Fermi-LAT data in an extended region of the galactic center $[239,240]$, showing a peak in the photon spectrum at an energy $\sim 130 \mathrm{GeV}$ with significance of the excess at $\sim 3 \sigma$ level. After an optimal observational strategy has been carried out for the purpose [241, 242], the updated analysis from the Fermi-LAT collaboration does not support any longer the original evidence for such a spectral feature in the dataset [243], suggesting previous claims to be related only to a statistical fluke. While current lack of detection of gamma-ray lines place important upper limits on today's DM annihilation cross section/decay rate into monochromatic photons in the GeV-TeV energy window [243, 244], the search for pronounced spectral features in the gamma-ray sky remains one of the most tantalizing observational programs within the WIMP mass reach [27].

Interestingly enough, within almost an entire decade, an increasing number of studies focused on the GC region, repeatedly showing the existence of a statistically significant signal in Fermi-LAT data possibly correlated with spectral and morphological features of prompt gamma-ray emission from DM pair annihilation [245-263]. Originally, the analysis of Fermi-LAT data in 2009 concerning the innermost two degrees around the GC leads the authors of $[247,248]$ to discover the existence of a bump-like feature in the photon spectrum exceeding the estimated astrophysical background with high statistical significance. Peaking around $\sim 3 \mathrm{GeV}$ and fitting an approximately spherical morphology, the signal found could be immediately associated with annihilating DM particles with mass range and cross section remarkably within the WIMP ballpark. Strengthening such compelling interpretation [252] and successively [254] focused on a region of interest (ROI) extended up to ten degrees in latitude, in correspondence to the low-latitude part of the Fermi bubbles [264], finding new evidence for the gammaray excess even at few $\mathrm{kpc}$ of distance from the GC. Eventually, in [257] an optimized analysis implementing specific cuts to Fermi-LAT events-improving the resolution of the gamma-ray maps - could reach a statistical preference at the $\sim 30 \sigma$ level for the inclusion of a WIMP-like template in the fit to the dataset. These claims triggered several phenomenological studies on the New Physics direction to undertake in order to explain this anomaly (see, e.g., [265272]).

It is important to note that a close investigation about the impact of the galactic diffuse emission-related to CR physics-on the robustness of the aforementioned evidence in favor of a DM indirect detection came only four years after [247], with the studies in [258, 259]. In particular, [259] analyzed the uncertainties related to the galactic diffuse modeling explicitly inspecting the systematics of 60 models with different characterization of CR transport physics and emitting target properties. Most importantly, the authors in [259] derived in a more data-driven way the overall systematics in the ROI of $20^{\circ} \times 20^{\circ}$ centered at the GC by looking at the residuals obtained from a large number of control ROI along the whole galactic plane, using their 60 galactic diffuse emission models for a principal component analysis. The systematics found in [259] associated with the GC excess signal enlarged the set of viable DM best-fit scenarios [269, 270] and opened a new window for the interpretation of the anomaly in terms of a population of point sources such as millisecond pulsars (MPs) [273], a scenario originally proposed in [274] and successively supported in [251, 253, $256,275]$, while criticized in $[276,277]$. 
Alternative astrophysical explanations in terms of outburst events of hadronic [278] or leptonic [279] origin turned out to be not favored by data [280]. On the other hand, a currently viable alternative scenario for the $\mathrm{GeV}$ gammaray spectral feature may be provided by the depletion of low-energy photons in molecular clouds as observed in the central molecular zone [263] (see also, for comparison, the sophisticated data-analysis and deconvolution technique presented in [281]).

Notably, the different $C R$ injection terms $\left(Q_{0}\right.$ appearing in (10)) adopted in all the aforementioned analyses correspond to radial distributions that in the GC proximity do not correlate well with the expected high-star formation rate present in the inmost few hundred parsecs around the GC [282], the so-called central molecular zone (CMZ). Multiwavelength observations of the CMZ [283] point indeed to an environment with large amount of molecular gas [284], hosting highmass OB stars [285], and potential progenitors for standard CR acceleration sites such as supernova remnants [286]. These pieces of information motivated [245] to reanalyze the GC excess implementing a novel steady-state CR source term capturing the CMZ star-forming activity. The three panels in Figure 4 from [245] show the residual count maps of photons falling in the energy range of $1-10 \mathrm{GeV}$, within a ROI of $10^{\circ} \times 10^{\circ}$ centered at the GC. From left to right, the result within a benchmark scenario originally identified in [259] to optimize the study of the GC anomaly at the GeV and the remarkable improvement in the outcome of the fit to Fermi-LAT data when adding a DM component (central panel) or when revisiting the whole galactic diffuse emission background on the basis of the novel steady source term peaked at the GC (right panel).

This result has been successively corroborated and refined in $[260,287]$, with the implementation of the high-resolution galactic gas distribution obtained in [223], able to resolve spiral arms and the galactic bar, and most importantly providing kinematic resolution towards the GC, with the help of hydrodynamic simulations. These HII density maps have been consequently correlated to the CR source injection term by means of a simple model of star formation; see [288], in order to provide an improved modeling of CR physics at the GC.

The relevance of the galactic diffuse emission modeling when assessing the significance of the $\mathrm{GeV}$ excess at the GC has been further acknowledged by the Fermi-LAT collaboration [289]. In [289], injected CR electrons as in [260], together with the uncertainties on the interstellar radiation field, have been shown to play a major role in the characterization of the gamma-ray anomaly. As also marked by the right-bottom panel of Figure 4, noisy residual photon counts around the GC in the analyses of $[245,260,289]$ still leave room for an extra component in the description of gamma-ray data. Notably, two independent studies implementing different statistical techniques for clustering patterns in the observed photon count maps have followed, showing very high evidence in favor of a hitherto undetected population of point sources, able to fully account for the $\mathrm{GeV}$ gamma-ray excess $[290,291]$. The results of these two works reached remarkably similar conclusions, giving substantial credit to the
MSP-like interpretation of the gamma-ray signal at the GC (see $[292,293]$ for discussions about the luminosity function of the putative MSPs at the GC) and triggering relevant dedicated searches [294]. Nonnegligible mismodeling of astrophysical backgrounds and foregrounds may affect the details on the prediction for such unresolved population of point sources within the galactic bulge; see, e.g., [261]. A recent novel tool, SkyFACT, developed in [295], based on image reconstruction and adaptive spatiospectral template regression, has allowed for dramatic improvements in the quality of the fits to gamma-ray data through fine-grained variations of galactic diffuse emission modeling. Exploiting this powerful package, a novel investigation on the morphology of the excess in connection with the stellar distribution of the bar/boxy bulge in the inner galaxy provides strong support to the MSP hypothesis [296].

In conclusion, the GC GeV excess remains a widely studied signal in the astro-particle community. The updated comprehensive analysis carried out by the Fermi-LAT collaboration [262] fairly summarizes the most relevant factors affecting the characterization of the signal: (1) the details on $\mathrm{CR}$ production and propagation, especially in the GC proximity; (2) the templates for the interstellar gas and radiation field in the inner galaxy; (3) the emissivity and morphology of the Fermi bubbles towards low latitudes; (4) the list of point sources near the GC identified within a given background model.

The take-home message of this long debate is likely twofold: the existence of an extended emission from the inner galaxy peaked at few $\mathrm{GeV}$ is well established; however, the characterization of this emission, i.e., the morphology and its intensity, strongly depends on the assumptions of the CR source distribution (and probably also on the details of the interactions with the molecular environments). Interestingly, the interpretation in terms of unresolved point sources, possibly associated with a population of millisecond pulsars-currently supported by wavelet and photon count statistics analyses-is testable in the future with more sensitive radio facilities [297]. As a final outlook, let us mention that a recent spatially extended gamma-ray signal from the center of M31 galaxy [298] has further renewed the interest on the GC anomaly and, for instance, may possibly shed new light on the existence of galactic bulge MSP populations [299].

Let us now move away from the GC region and consider another very relevant potential discovery window. Contrary to the complex astrophysical environment characterizing the CMZ and the GC, dwarf spheroidal galaxies of the Milky Way (dSphs) stand out as very promising targets in the gamma-ray band due to the corresponding low astrophysical background and foreground $[300,301]$. Being relatively close to us and associated with fairly large DM densities, the gamma-ray campaign on dSphs has been soon realized to be one of the most potentially sensitive probes to particle DM properties [302-304]. At present, dSph gamma-ray upper-bounds are remarkably probing the benchmark thermal relic scenario within the WIMP mass window [305-308]. Such upper limits may be at odds with naive DM interpretations of the GC excess $[309,310]$, while depending crucially on the estimated 

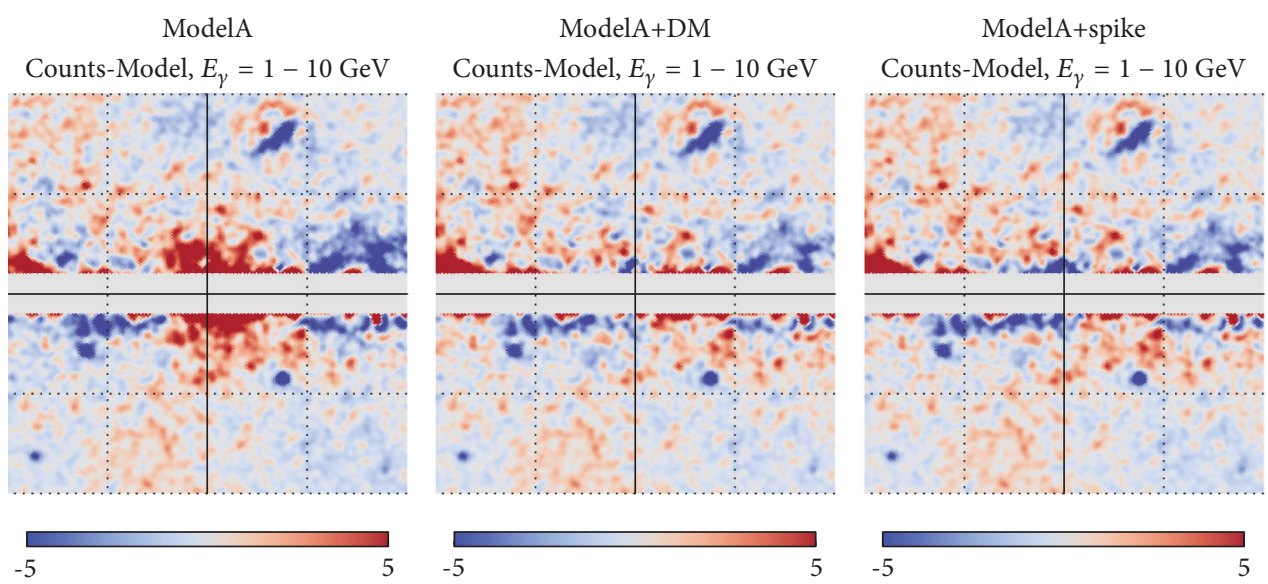

FIGURE 4: Importance of the cosmic-ray contribution to the galactic center excess at few $\mathrm{GeV}$ from the analysis of Fermi-LAT data. The three panels refer to the residual photon count maps discussed in [245]. Figure 4 is reproduced from Gaggero et al. (2015) [245] (under the Creative Commons Attribution License/public domain).

DM content in these galaxies, potentially affected by several systematics [311-314].

Interestingly, hints for a gamma-ray signal possibly compatible with the one observed at the GC have been found in the analysis of some of the most recently discovered Milky Way ultra-faint satellites; see, e.g., the case of Reticulum II [315-317]. According to the latest joint analysis of FermiLAT and Dark Energy Survey collaborations, the significance of these excesses remains at present well below the $3 \sigma$ level [307]. Moreover, dedicated searches in the radio-band have not found any counterpart of the putative gamma-ray DM signal [318]. Note that-from the perspective of a signal detection - a broad multiwavelength program for indirect DM searches in $\mathrm{d} S$ phs would be indeed promising $[319,320]$. However, in opposition to the case of gamma rays, constraints on DM annihilation/decay derived from the observation of $\mathrm{dSphs}$ in the radio and/or X band turn out to be subject to a larger set of astrophysical uncertainties, including CR transport physics [321].

\section{Future Prospects: From MeV to Multi-TeV}

The future of indirect searches is particularly bright.

In the gamma-ray band, two new regions of the spectrum will be explored. On the low-energy side, the $\mathrm{MeV}-\mathrm{GeV}$ domain can be probed by planned experiments such as eASTROGAM [322] $]^{7}$ and AMEGO ${ }^{8}$ (other previously proposed missions include, e.g., COMPAIR [323] and ADEPT [324]), which could be realized in the mid- and long-term future in the late 2020s). All those experiments will feature a 2 -3-order-of-magnitude increase in sensitivity $[325,326]$ with respect to previous instruments operating in this window like COMPTEL and EGRET [327, 328] and a remarkable energy resolution especially below $\simeq 10 \mathrm{GeV}$, where the detection principle is based on Compton scattering instead of pair production. This will guarantee a high constraining power as far as sharp spectral features (e.g., lines originating from DM annihilation) are concerned [329]. On theoretical grounds, vanilla WIMP scenario in this domain of energy scales may not be adequate due to the Lee-Weinberg bound [57, 58]. Interesting models designed to offer an explanation for a 511 $\mathrm{keV}$ emission line detected in the inner galaxy may be instead well probed [330, 331].

On the other end, in the $\mathrm{TeV}$ domain, while many Imaging Atmospheric Cherenkov Telescopes (MAGIC [332], HESS $^{9}$, and VERITAS ${ }^{10}$ ) and air-shower arrays (such as Milagro $^{11}$ and HAWC ${ }^{12}$ [333]) have already been providing relevant results and have provided stringent constraints on DM annihilation [334], CTA will provide a further, unprecedented increase in sensitivity above the $\mathrm{TeV}$. We want to emphasize that, as mentioned in Section 2, the most naive version of the WIMP miracle naturally yields a predicted mass for the DM candidate in that ballpark. CTA can probe the DM sector in different ways [335-338].

In connection with the main topic of this review, we stress once again that the GC region appears promising also in this context, which implies that a more careful modeling of the $\gamma$ ray diffuse emission from the inner galaxy will be required. The diffusion properties, again, play then a central role: in particular, the CR spectrum in the inner galaxy is currently a matter or debate, and Fermi-LAT data seem to hint towards a hardening in the inner galaxy [118, 229, 230]. DRAGON-based models $[116,118]$ featuring inhomogeneous or anisotropic diffusion, which are key features of this specific numerical package, allow reproducing Fermi-LAT data and providing $\mathrm{TeV}$ predictions $[119,228]$ which seem to be in accord with a collection of data from different experiments, including the bright and diffuse galactic Ridge emission measured by HESS over the latest decade [339-341]. These kind of studies will be even more important in the forthcoming years, due to the necessity, that we have stressed several times along this paper, of a good characterization of the astrophysical backgrounds. The study of dwarf spheroidal galaxies will also benefit from the expected increase in sensitivity in the $\mathrm{TeV}$ domain, and the expected performance of CTA in this channel is remarkable [342-344].

As far as charged particle channels are concerned, the antiparticle/antinuclear avenue is particularly promising 
despite the relevant uncertainties. In fact, new high-quality data are expected by many experiments: besides AMS-02, the already operating DAMPE [345] space experiment, CALET [346], on board the International Space Station, and the nextgeneration balloon ISS-CREAM [347] have the opportunity to probe in particular the leptonic channel all the way up to the multi- $\mathrm{TeV}$ range with unprecedented energy resolution and sensitivity; planned experiments such as $\mathrm{HERD}^{13}$ will provide a further very relevant extension in the covered energy range and sensitivity, and-on the antinuclei side-we are looking forward to experiments such as GAPS [348], a balloon experiment expected to operate in 2020 and optimized specifically for low-energy antinuclei signatures, thanks to a novel detection technique based on exotic atom capture and decay.

To conclude this grand tour, let us emphasize once again the opportunity offered by the tremendous increase in experimental accuracy we are already witnessing in these days, and we will be further exploiting with the upcoming years. In our opinion, in order to take full advantage of these developments, interdisciplinarity is the main avenue. The guaranteed outcome of the research program aimed at indirectly detecting particle dark matter by means of astronomical data certainly involves a more profound understanding of the CR physics outlined across this review. It is therefore crucial to deepen and broaden the connections between the research fields we have described above: both the high energy astrophysics and the particle physics community would unquestionably benefit from a cutting-edge increasing crossover.

\section{Conflicts of Interest}

The authors declare that there are no conflicts of interest regarding the publication of this article.

\section{Acknowledgments}

Thanks are due to Dario Grasso, Gianfranco Bertone, Piero Ullio, and Stefano Gabici for all their invaluable teachings about dark matter and cosmic-ray physics. The authors thank Alfredo Urbano and Carmelo Evoli for batting around a few interesting ideas on how to improve the manuscript.

\section{Endnotes}

1. The Alfvén speed is supersonic in most typical ISM environments: hot $\mathrm{HII}$ regions, warm intercloud gas, and molecular gas, with $\beta \equiv\left(v_{s} / v_{A}\right)^{2} \simeq 0.1 \div 0.3$ everywhere [349].

2. As a reference, the reader can keep in mind that the energy scales from $\mathrm{GeV}$ to $\mathrm{PeV}$, characteristic of galactic $\mathrm{CRs}$, resonate with scales from $\mathscr{O}(\mathrm{AU})$ to $\mathscr{O}(\mathrm{pc})$.

3. Equivalently, in term of pitch angle, in the direction along the regular field we can write

$$
\frac{\partial f}{\partial t}+v \mu \frac{\partial f}{\partial x}=\frac{\partial}{\partial \mu}\left(D_{\mu \mu} \frac{\partial f}{\partial \mu}\right) .
$$

4. We outline in particular the relevant role of the crosssection uncertainties (see also [135] and references therein). In this regard, there has been a remarkable activity in the latest years both concerning semiempirical parametrizations (tuned on experimental datasets) and Monte Carlo event generators: as far as the former category is concerned, new models have been proposed based on the data provided by the NA49 and BRAHMS collaborations [350-353]; concerning the latter, several codes (e.g., EPOS 1.99 [354], SIBYLL [355], and QGSJET-II-04 [356]) have been recently tuned to LHC data (see, e.g., $[354,356])$.

5. http://dpnc.unige.ch/dampe/.

6. http://calet.phys.lsu.edu/.

7. e-ASTROGAM is proposed as ESA M5 mission.

8. See pcos.gsfc.nasa.gov/physpag/probe/AMEGO_probe .pdf

9. www.mpi-hd.mpg.de/hfm/HESS.

10. veritas.sao.arizona.edu/.

11. http://umdgrb.umd.edu/cosmic/milagro.html.

12. umdgrb.umd.edu/hawc/index.php.

13. http://herd.ihep.ac.cn/.

\section{References}

[1] J. Silk et al., Particle Dark Matter: Observations, Models and Searches, Cambridge University Press, 2010, http://www.cambridge.org/uk/catalogue/catalogue. asp?isbn=9780521763684.

[2] J. Abdallah et al., "Simplified Models for Dark Matter Searches at the LHC," Physics of the Dark Universe, vol. 9-10, pp. 8-23, 2015.

[3] T. Marrodan Undagoitia and L. Rauch, "Dark matter directdetection experiments," Journal of Physics G: Nuclear and Particle Physics, vol. 43, 2016.

[4] J. Ellis, J. S. Hagelin, D. V. Nanopoulos, K. Olive, and M. Srednicki, "Supersymmetric relics from the big bang," Nuclear Physics B, vol. 238, no. 2, pp. 453-476, 1984.

[5] G. Jungman, M. Kamionkowski, and K. Griest, "Supersymmetric dark matter," Physics Reports, vol. 267, pp. 195-373, 1996.

[6] K. A. Olive, "Lectures on Dark Matter," in Proceedings of Particle Physics and Cosmology: The Quest for Physics beyond the Standard Model(s), TASI 2002, June 3-28, 2002, pp. 797-851, Theoretical Advanced Study Institute, Boulder, Colorado, USA, 2003.

[7] Y. B. Zeldovich, A. A. Klypin, M. Y. Khlopov, and V. M. Chechetkin, "Astrophysical constraints on the mass of heavy stable neutral leptons," Soviet Journal of Nuclear Physics, vol. 31, pp. 664-669, 1980, Yad.Fiz. 31 (1980) 1286-1294.

[8] D. Maurin, R. Taillet, F. Donato, P. Salati, A. Barrau, and G. Boudoul, "Galactic Cosmic Ray Nuclei as a Tool for Astroparticle Physics," Recent Research Developments in Astrophysics, 2002.

[9] L. E. Strigari, "Galactic Searches for Dark Matter," Physics Reports, vol. 531, no. 1, 2013.

[10] L. Bergstrom, "Non-baryonic dark matter - observational evidence and detection methods," Reports on Progress in Physics, vol. 63, p. 793, 2000. 
[11] G. Bertone, D. Hooper, and J. Silk, "Particle dark matter: evidence, candidates and constraints," Physics Reports, vol. 405, pp. 279-390, 2005.

[12] J. Silk and M. Srednicki, "Cosmic-Ray Antiprotons as a Probe of a Photino-Dominated Universe," Physical Review Letters, vol. 53, p. 624, 1984.

[13] F. W. Stecker, S. Rudaz, and T. F. Walsh, "Galactic Antiprotons from Photinos," Physical Review Letters, vol. 55, p. 2622, 1985.

[14] P. Picozza et al., "PAMELA - a payload for antimatter matter exploration and light-nuclei astrophysics," Astroparticle Physics, vol. 27, pp. 296-315, 2007.

[15] A. Kounine, "The alpha magnetic spectrometer on the international space station," International Journal of Modern Physics E, vol. 21, 2012.

[16] A. Reinert and M. W. Winkler, "A precision search for WIMPs with charged cosmic rays," Journal of Cosmology and Astroparticle Physics, 2017.

[17] K. Blum, R. Sato, and E. Waxman, Cosmic-ray Antimatter, 2017, http://inspirehep.net/record/1624395.

[18] O. Adriani, G. C. Barbarino, and G. A. Bazilevskaya, "An anomalous positron abundance in cosmic rays with energies 1.5-100 GeV," Nature, vol. 458, pp. 607-609, 2009.

[19] M. Aguilar, G. Alberti, B. Alpat et al., "First Result from the Alpha Magnetic Spectrometer on the International Space Station: Precision Measurement of the Positron Fraction in Primary Cosmic Rays of 0.5-350 GeV," Physical Review Letters, vol. 110, 2013.

[20] M. Aguilar, L. Ali Cavasonza, B. Alpat et al., "Antiproton flux, antiproton-to-proton flux ratio, and properties of elementary particle fluxes in primary cosmic rays measured with the alpha magnetic spectrometer on the international space station," Physical Review Letters, vol. 117, 2016.

[21] A. Cuoco, M. Krämer, and M. Korsmeier, "Novel dark matter constraints from antiprotons in light of AMS-02," Physical Review Letters, vol. 118, 2017.

[22] A. Cuoco, J. Heisig, M. Korsmeier, and M. Kramer, "A combined dark matter study of AMS-02 antiprotons and Fermi-LAT gamma rays," in Proceedings of the 2017 European Physical Society Conference on High Energy Physics (EPS-HEP 2017), Venice, Italy, July 5-12, 2017.

[23] L. Bergström and H. Snellman, "Observable monochromatic photons from cosmic photino annihilation," Physical Review D, vol. 37, 1988.

[24] S. Rudaz, "Annihilation of heavy-neutral-fermion pairs into monochromatic $\gamma$ rays and its astrophysical implications," Physical Review D, vol. 39, 1989.

[25] G. F. Giudice and K. Griest, "Rate for annihilation of galactic dark matter into two photons," Physical Review D, vol. 2549, 1989.

[26] L. Bergström, P. Ullio, and J. H. Buckley, "Observability of gamma rays from dark matter neutralino annihilations in the milky way halo," Astroparticle Physics, vol. 9, pp. 137-162, 1998.

[27] T. Bringmann and C. Weniger, "Gamma ray signals from dark matter: Concepts, status and prospects," Physics of the Dark Universe, vol. 1, pp. 194-217, 2012.

[28] G. Bertone and D. Hooper, "A history of dark matter," Reviews of Modern Physics, 2016.

[29] J. de Swart, G. Bertone, and J. van Dongen, "How dark matter came to matter," Nature Astronomy, 2017.

[30] W. Hu, R. Barkana, and A. Gruzinov, "Fuzzy Cold Dark Matter: The Wave Properties of Ultralight Particles," Physical Review Letters, vol. 85, 2000.
[31] D. J. E. Marsh, "Axion cosmology," Physics Reports, vol. 643, pp. 1-79, 2016.

[32] L. Hui, J. P. Ostriker, S. Tremaine, and E. Witten, "Ultralight scalars as cosmological dark matter," Physical Review D, vol. 95, 2017.

[33] P. Svrvcek and E. Witten, "Axions in string theory," Journal of High Energy Physics, vol. 6, article no. 51, 2006.

[34] A. Arvanitaki, S. Dimopoulos, S. Dubovsky, N. Kaloper, and J. March-Russell, "String axiverse," Physical Review D, vol. 81, 2010.

[35] B. P. Abbott et al., "Observation of Gravitational Waves from a Binary Black Hole Merger," Physical Review Letters, vol. 116, 2016.

[36] S. Bird, I. Cholis, J. B. Muñoz et al., "Did LIGO detect dark matter?" Physical Review Letters, vol. 116, 2016.

[37] G. F. Chapline, "Cosmological effects of primordial black holes," Nature (London), vol. 251, pp. 251-252, 1975.

[38] B. Carr, F. Kühnel, and M. Sandstad, "Primordial black holes as dark matter," Physical Review D, vol. 94, 2016.

[39] J. L. Feng, "Dark matter candidates from particle physics and methods of detection," Annual Review of Astronomy and Astrophysics, vol. 48, pp. 495-545, 2010.

[40] G. F. Giudice, "Naturally speaking: the naturalness criterion and physics at the LHC," in Perspectives on LHC Physics, 2008.

[41] M. Regis, M. Serone, and P. Ullio, "A dark matter candidate from an extra (Non-Universal) dimension," Journal of High Energy Physics, vol. 03, article no. 84, 2007.

[42] G. Panico, E. Pontón, J. Santiago, and M. Serone, "Dark matter and electroweak symmetry breaking in models with warped extra dimensions," Physical Review D, vol. 77, 2008.

[43] T. A. Ryttov and F. Sannino, "Ultraminimal technicolor and its dark matter technicolor interacting massive particles," Physical Review D, vol. 78, 2008.

[44] A. Belyaev, M. T. Frandsen, S. Sarkar, and F. Sannino, "Mixed dark matter from technicolor," Physical Review D, vol. 83, 2011.

[45] M. Frigerio, A. Pomarol, F. Riva, and A. Urbano, "Composite scalar dark matter," Journal of High Energy Physics, vol. 7, article no. 15, 2012.

[46] S. Bruggisser, F. Riva, and A. Urbano, "Strongly interacting light dark matter," SciPost Physics, vol. 3, no. 17, 2017.

[47] G. F. Giudice and A. Romanino, "Split Supersymmetry," Nuclear Physics B, vol. 699, pp. 65-89, 2004, Erratum to: "Split Supersymmetry," Nuclear Physics B, vol. 706, p. 487, 2005.

[48] N. Arkani-Hamed, S. Dimopoulos, G. F. Giudice, and A. Romanino, "Aspects of Split Supersymmetry," Nuclear Physics $B$, vol. 709, pp. 3-46, 2005.

[49] A. Masiero, S. Profumo, and P. Ullio, "Neutralino dark matter detection in split supersymmetry scenarios," Nuclear Physics B, vol. 712, pp. 86-114, 2005.

[50] G. Servant and T. M. P. Tait, "Is the lightest kaluza-klein particle a viable dark matter candidate?" Nuclear Physics B, vol. 650, pp. 391-419, 2003.

[51] K. Agashe and G. Servant, "Warped unification, proton stability and dark matter," Physical Review Letters, vol. 93, 2004.

[52] K. Agashe and G. Servant, "Baryon number in warped grand unified theories: model building and (dark matter related) phenomenology," Journal of Cosmology and Astroparticle Physics, vol. 2005, no. 02, 2005.

[53] D. Hooper and S. Profumo, "Dark matter and collider phenomenology of universal extra dimensions," Physics Reports, vol. 453, pp. 29-115, 2007. 
[54] P. Gondolo and G. Gelmini, "Cosmic abundances of stable particles: Improved analysis," Nuclear Physics B, vol. 360, no. 1, pp. 145-179, 1991.

[55] S. Profumo, An Introduction to Particle Dark Matter, World Scientific, 2017.

[56] P. A. R. Ade et al., "Planck 2015 results. XIII. Cosmological parameters," Astronomy and Astrophysics, vol. 594, no. A13, 2016.

[57] M. I. Vysotsky, A. D. Dolgov, and Y. B. Zeldovich, "Cosmological limits on the masses of neutral leptons," JETP Letters, vol. 26, p. 188, 1977.

[58] B. W. Lee and S. Weinberg, "Cosmological lower bound on heavy-neutrino masses," Physical Review Letters, vol. 39, no. 4, 1977.

[59] K. Griest and M. Kamionkowski, "Unitarity limits on the mass and radius of dark-matter particles," Physical Review Letters, vol. 64, no. 6, 1990.

[60] K. Harigaya, M. Ibe, K. Kaneta, W. Nakano, and M. Suzuki, "Thermal relic dark matter beyond the unitarity limit," Journal of High Energy Physics, vol. 2016, no. 8, 2016.

[61] K. Griest and D. Seckel, "Three exceptions in the calculation of relic abundances," Physical Review D: Particles, Fields, Gravitation and Cosmology, vol. 43, no. 10, 1991.

[62] E. D. Carlson, M. E. Machacek, and L. J. Hall, "Self-interacting dark matter," The Astrophysical Journal, vol. 398, pp. 43-52, 1992.

[63] M. Pospelov, A. Ritz, and M. Voloshin, "Secluded WIMP dark matter," Physics Letters B, vol. 662, no. 1, pp. 53-61, 2008.

[64] Y. Hochberg, E. Kuflik, T. Volansky, and J. G. Wacker, "Mechanism for thermal relic dark matter of strongly interacting massive particles," Physical Review Letters, vol. 113, no. 17, 2014.

[65] F. D'Eramo and J. Thaler, "Semi-annihilation of dark matter," Journal of High Energy Physics, vol. 2010, no. 06, 2010.

[66] R. T. D’Agnolo and J. T. Ruderman, "Light dark matter from forbidden channels," Physical Review Letters, vol. 115, no. 6, 2015.

[67] D. Pappadopulo, J. T. Ruderman, and G. Trevisan, "Dark matter freeze-out in a nonrelativistic sector," Physical Review D: Particles, Fields, Gravitation and Cosmology, vol. 94, no. 3, 2016.

[68] J. A. Dror, E. Kuflik, and W. H. Ng, "Codecaying dark matter," Physical Review Letters, vol. 117, no. 21, 2016.

[69] R. T. D'Agnolo, D. Pappadopulo, and J. T. Ruderman, "Fourth exception in the calculation of relic abundances," Physical Review Letters, vol. 119, no. 6, 2017.

[70] F. D’Eramo, N. Fernandez, and S. Profumo, "When the universe expands too fast: relentless dark matter," Journal of Cosmology and Astroparticle Physics, vol. 2017, no. 5, 2017.

[71] N. Arkani-Hamed, D. P. Finkbeiner, T. R. Slatyer, and N. Weiner, "A theory of dark matter," Physical Review D: Particles, Fields, Gravitation and Cosmology, vol. 79, no. 1, 2009.

[72] J. L. Feng, M. Kaplinghat, and H.-B. Yu, "Halo-shape and relic-density exclusions of sommerfeld-enhanced dark matter explanations of cosmic ray excesses," Physical Review Letters, vol. 104, no. 15, 2010.

[73] J. L. Feng, M. Kaplinghat, and H.-B. Yu, "Sommerfeld enhancements for thermal relic dark matter," Physical Review D: Particles, Fields, Gravitation and Cosmology, vol. 82, no. 8, 2010.

[74] A. Hryczuk, R. Iengo, and P. Ullio, "Relic densities including Sommerfeld enhancements in the MSSM," Journal of High Energy Physics, vol. 2011, no. 3, 2011.

[75] W. Shepherd, T. M. Tait, and G. Zaharijas, "Bound states of weakly interacting dark matter," Physical Review D: Particles, Fields, Gravitation and Cosmology, vol. 79, no. 5, 2009.
[76] B. von Harling and K. Petraki, "Bound-state formation for thermal relic dark matter and unitarity," Journal of Cosmology and Astroparticle Physics, vol. 2014, no. 12, 2014.

[77] H. An, M. B. Wise, and Y. Zhang, "Effects of bound states on dark matter annihilation," Physical Review D, vol. 93, no. 11, 2016.

[78] M. Cirelli, P. Panci, K. Petraki, F. Sala, and M. Taoso, "Dark Matter's secret liaisons: phenomenology of a dark U(1) sector with bound states," Journal of Cosmology and Astroparticle Physics, vol. 2017, no. 05, 2017.

[79] A. Mitridate, M. Redi, J. Smirnov, and A. Strumia, "Cosmological implications of dark matter bound states," Journal of Cosmology and Astroparticle Physics, vol. 2017, no. 05, 2017.

[80] S. Biondini and M. Laine, "Thermal dark matter co-annihilating with a strongly interacting scalar," Journal of High Energy Physics, vol. 2018, no. 4, 2018.

[81] J. L. Feng and J. Kumar, "Dark-Matter particles without weakscale masses or weak interactions," Physical Review Letters, vol. 101, no. 23, 2008.

[82] M. Cirelli, G. Corcella, A. Hektor et al., "PPPC 4 DM ID: a poor particle physicist cookbook for dark matter indirect detection," Journal of Cosmology and Astroparticle Physics, vol. 2011, no. 03, 2011, Erratum: "PPPC 4 DM ID: a poor particle physicist cookbook for dark matter indirect detection," Journal of Cosmology and Astroparticle Physics, vol. 2012, no. 10, 2012.

[83] J. Buch, M. Cirelli, G. Giesen, and M. Taoso, "PPPC 4 DM secondary: a Poor Particle Physicist Cookbook for secondary radiation from Dark Matter," Journal of Cosmology and Astroparticle Physics, vol. 2015, no. 09, 2015.

[84] G. Arcadi, M. Dutra, P. Ghosh et al., "The waning of the WIMP? A review of models, searches, and constraints," The European Physical Journal C, 2017.

[85] A. R. Bell, "Cosmic ray acceleration," Astroparticle Physics, vol. 43, pp. 56-70, 2013.

[86] W. Baade and F. Zwicky, Contributions from the Mount Wilson Observatory, vol. 3, 1934.

[87] W. Baade and F. Zwicky, "Remarks on super-novae and cosmic rays," Physical Review, vol. 46, no. 1, 1934.

[88] P. Morrison, "On the origins of cosmic rays," Reviews of Modern Physics, vol. 29, 1957.

[89] V. L. Ginzburg, "The nature of cosmic radio emission and the origin of cosmic rays," Il Nuovo Cimento, vol. 3, pp. 38-48, 1956.

[90] R. D. Blandford and J. P. Ostriker, "Particle acceleration by astrophysical shocks," The Astrophysical Journal Letters, vol. 221, pp. L29-L32, 1978.

[91] A. R. Bell, "The acceleration of cosmic rays in shock fronts. I," Monthly Notices of the Royal Astronomical Society, vol. 182, pp. 147-156, 1978.

[92] W. I. Axford, E. Leer, and G. Skadron, "The acceleration of cosmic rays by shock waves," in Proceedings of the 15th International Cosmic Ray Conference, vol. 11, pp. 132-137, 1977.

[93] G. F. Krymskii, "A regular mechanism for the acceleration of charged particles on the front of a shock wave," Akademiia Nauk SSSR Doklady, vol. 234, pp. 1306-1308, 1977.

[94] F. A. Aharonian, A. M. Atoyan, and H. J. Voelk, "High energy electrons and positrons in cosmic rays as an indicator of the existence of a nearby cosmic tevatron," Astronomy and Astrophysics, vol. 294, pp. L41-L44, 1995.

[95] R. P. Murphy, M. Sasaki, W. R. Binns et al., "Galactic cosmic ray origins and $\mathrm{OB}$ associations: evidence from SuperTIGER 
observations of elements ${ }_{26} \mathrm{Fe}$ through ${ }_{40} \mathrm{Zr}$," The Astrophysical Journal, vol. 831, 2016.

[96] S. Heinz and R. Sunyaev, "Cosmic rays from microquasars: A narrow component to the CR spectrum?" Astronomy and Astrophysics, vol. 390, no. 2, pp. 751-766, 2002.

[97] T. Antoni, W. D. Apel, A. F. Badea et al., "Large-Scale CosmicRay Anisotropy with KASCADE," The Astrophysical Journal, vol. 604, no. 2, pp. 687-692, 2004.

[98] A. A. Abdo, B. T. Allen, T. Aune et al., "The large-scale cosmicray anisotropy as observed with milagro," The Astrophysical Journal, vol. 698, no. 2, pp. 2121-2130, 2009.

[99] M. Aglietta, V. V. Alekseenko, B. Alessandro et al., "Evolution of the cosmic-ray anisotropy above $10^{14} \mathrm{eV}$," Astrophysical Journal Letters, vol. 692, no. 2, pp. L130-L133, 2009.

[100] M. Amenomori, X. J. Bi, D. Chen et al., "Northern sky galactic cosmic ray anisotropy between 10 and $1000 \mathrm{TeV}$ with the tibet air shower array," The Astrophysical Journal, vol. 836, no. 2, 2017.

[101] M. G. Aartsen, R. Abbasi, Y. Abdou et al., "Observation of cosmic-ray anisotropy with the icetop air shower array," The Astrophysical Journal, vol. 765, no. 1, 2013.

[102] R. Abbasi, Y. Abdou, T. Abu-Zayyad et al., "Observation of anisotropy in the galactic cosmic-ray arrival directions at 400 $\mathrm{TeV}$ with icecube," The Astrophysical Journal, vol. 746, no. 1, 2012.

[103] J. B. Pollack and G. G. Fazio, "Production of $\pi$ mesons and gamma radiation in the galaxy by cosmic rays," Physical Review, vol. 131, no. 6, pp. 2684-2691, 1963.

[104] V. L. Ginzburg and S. I. Syrovatskii, The Origin of Cosmic Rays, Elsevier, 1964.

[105] V. S. Berezinskii, S. V. Bulanov, V. A. Dogiel, and V. S. Ptuskin, Astrophysics of Cosmic Rays, North-Holland, 1990.

[106] J. R. Jokipii, "Cosmic-ray propagation. I. Charged particles in a random magnetic field," The Astrophysical Journal, vol. 146, 1966.

[107] J. R. Jokipii and E. N. Parker, "Random walk of magnetic lines of force in astrophysics," Physical Review Letters, vol. 21, no. 1, pp. 44-47, 1968.

[108] J. W. Armstrong, B. J. Rickett, and S. R. Spangler, "Electron density power spectrum in the local interstellar medium," The Astrophysical Journal, vol. 443, no. 1, pp. 209-221, 1995.

[109] R. Jansson and G. R. Farrar, "The galactic magnetic field," The Astrophysical Journal Letters, vol. 761, no. 1, 2012.

[110] P. Terral and K. Ferrière, "Constraints from Faraday rotation on the magnetic field structure in the Galactic halo," Astronomy \& Astrophysics, vol. 600, 2017.

[111] B. G. Elmegreen and J. Scalo, "Interstellar turbulence I: Observations and processes," Annual Review of Astronomy and Astrophysics, vol. 42, pp. 211-273, 2004.

[112] C. J. Cesarsky, "Cosmic-ray confinement in the galaxy," Annual Review of Astronomy and Astrophysics, vol. 18, pp. 289-319, 1980.

[113] P. Blasi, E. Amato, and P. D. Serpico, "Spectral breaks as a signature of cosmic ray induced turbulence in the galaxy," Physical Review Letters, vol. 109, no. 6, 2012.

[114] P. Blasi, "The origin of galactic cosmic rays," Astronomy and Astrophysics Reviews, vol. 21, no. 70, 2013.

[115] J. Skilling, "Cosmic ray streaming-I effect of Alfvén waves on particles," Monthly Notices of the Royal Astronomical Society, vol. 172 , no. 3, pp. 557-566, 1975.

[116] S. S. Cerri, D. Gaggero, A. Vittino, C. Evoli, and D. Grasso, "A signature of anisotropic cosmic-ray transport in the gamma-ray sky," Journal of Cosmology and Astroparticle Physics, vol. 2017, no. 10, 2017.

[117] C. Evoli, D. Gaggero, D. Grasso, and L. Maccione, "Common solution to the cosmic ray anisotropy and gradient problems," Physical Review Letters, vol. 108, no. 21, 2012.

[118] D. Gaggero, M. Taoso, A. Urbano, M. Valli, and P. Ullio, "Gamma-ray sky points to radial gradients in cosmic-ray transport," Physical Review D, vol. 91, no. 8, 2015.

[119] D. Gaggero, D. Grasso, A. Marinelli, M. Taoso, and A. Urbano, "Diffuse cosmic rays shining in the Galactic center: A novel interpretation of H.E.S.S. and Fermi-LAT gamma-ray data," Physical Review Letters, vol. 119, 2017.

[120] N. Tomassetti, "Cosmic-ray protons, nuclei, electrons, and antiparticles under a two-halo scenario of diffusive propagation," Physical Review D, vol. 92, 2015.

[121] J. Feng, N. Tomassetti, and A. Oliva, "Bayesian analysis of spatial-dependent cosmic-ray propagation: astrophysical background of antiprotons and positrons," Physical Review D, vol. 94, no. 12, 2016.

[122] A. W. Strong, I. V. Moskalenko, and V. S. Ptuskin, "Cosmic-ray propagation and interactions in the galaxy," Annual Review of Nuclear and Particle Science, vol. 57, pp. 285-327, 2007.

[123] C. Evoli, D. Gaggero, A. Vittino et al., "Cosmic-ray propagation with DRAGON2: I. Numerical solver and astrophysical ingredients," Journal of Cosmology and Astroparticle Physics, vol. 2017, no. 2, article no. 015, 2017.

[124] S. Sridhar and P. Goldreich, "Toward a theory of interstellar turbulence. I. Weak Alfvénic turbulence," The Astrophysical Journal, vol. 432, no. 2, pp. 612-621, 1994.

[125] P. Goldreich and S. Sridhar, "Toward a theory of interstellar turbulence. II. Strong Alfvénic turbulence," The Astrophysical Journal, vol. 438, no. 2, pp. 763-775, 1995.

[126] B. D. G. Chandran, "Scattering of energetic particles by anisotropic magnetohydrodynamic turbulence with a goldreich-sridhar power spectrum," Physical Review Letters, vol. 85, no. 22, 2000.

[127] H. Yan and A. Lazarian, "Scattering of cosmic rays by magnetohydrodynamic interstellar turbulence," Physical Review Letters, vol. 89, no. 28, 2002.

[128] H. Yan and A. Lazarian, "Cosmic ray propagation: nonlinear diffusion parallel and perpendicular to mean magnetic field," The Astrophysical Journal, vol. 673, no. 2, 2008.

[129] A. W. Strong and G. Youssefi, "Propagation models for CR nucleons and electrons and predictions of the galactic gammaray spectrum," in Proceedings of the 24th International Cosmic Ray Conference, vol. 3, p. 48, 1995.

[130] A. W. Strong and I. V. Moskalenko, "Propagation of cosmic-ray nucleons in the galaxy," The Astrophysical Journal, vol. 509, no. $1,1998$.

[131] I. V. Moskalenko and A. W. Strong, "Production and propagation of cosmic-ray positrons and electrons," The Astrophysical Journal, vol. 493, no. 2, 1998.

[132] A. W. Strong and I. V. Moskalenko, "New developments in the GALPROP CR propagation model," in Proceedings of the 27th International Cosmic Ray Conference, vol. 5, pp. 1942-1945, 2001.

[133] C. Evoli, D. Gaggero, D. Grasso, and L. Maccione, "Cosmic-ray nuclei, antiprotons and gamma-rays in the galaxy: a new diffusion model," Journal of Cosmology and Astroparticle Physics, vol. 2008, no. 10, 2008.

[134] D. Gaggero, L. Maccione, G. Di Bernardo, C. Evoli, and D. Grasso, "Three-dimensional model of cosmic-ray lepton propagation reproduces data from the alpha magnetic spectrometer 
on the international space station," Physical Review Letters, vol. 111, no. 2, 2013.

[135] C. Evoli, D. Gaggero, A. Vittino, M. Di Mauro, D. Grasso, and M. N. Mazziotta, "Cosmic-ray propagation with DRAGON2: II. Nuclear interactions with the interstellar gas," Journal of Cosmology and Astroparticle Physics, vol. 2018, 2018.

[136] R. Kissmann, "PICARD: A novel code for the Galactic Cosmic Ray propagation problem," Astroparticle Physics, vol. 55, pp. 3750, 2014.

[137] M. Werner, R. Kissmann, A. W. Strong, and O. Reimer, "Spiral arms as cosmic ray source distributions," Astroparticle Physics, vol. 64, pp. 18-33, 2015.

[138] D. Maurin, F. Donato, R. Taillet, and P. Salati, "Cosmic rays below $\mathrm{Z}=30$ in a diffusion model: New constraints on propagation parameters," The Astrophysical Journal, vol. 555, no. 2, 2001.

[139] G. Giesen, M. Boudaud, Y. Génolini et al., "AMS-02 antiprotons, at last! Secondary astrophysical component and immediate implications for dark matter," Journal of Cosmology and Astroparticle Physics, vol. 2015, 2015.

[140] M. Aguilar, L. Ali Cavasonza, G. Ambrosi et al., "Precision measurement of the boron to carbon flux ratio in cosmic rays from $1.9 \mathrm{GV}$ to $2.6 \mathrm{TV}$ with the alpha magnetic spectrometer on the international space station," Physical Review Letters, vol. 117, no. 23, 2016.

[141] R. Trotta, G. Jóhannesson, I. V. Moskalenko, T. A. Porter, R. Ruiz De Austri, and A. W. Strong, "Constraints on cosmicray propagation models from a global bayesian analysis," The Astrophysical Journal, vol. 729, no. 2, 2011.

[142] G. Jóhannesson, R. R. de Austri, A. C. Vincent et al., "Bayesian analysis of cosmic ray propagation: evidence against homogeneous diffusion," The Astrophysical Journal, vol. 824, no. 1, 2016.

[143] C. Evoli, D. Gaggero, and D. Grasso, "Secondary antiprotons as a galactic dark matter probe," Journal of Cosmology and Astroparticle Physics, vol. 2015, no. 12, 2015.

[144] Q. Yuan, S.-J. Lin, K. Fang, and X.-J. Bi, "Propagation of cosmic rays in the AMS-02 era," Physical Review D, vol. 95, no. 8, 2017.

[145] J.-S. Niu and T. Li, "Galactic cosmic-ray model in the light of AMS-02 nuclei data," Physical Review D, vol. 97, no. 2, 2017.

[146] A. Buffington and S. M. Schindler, "Recent cosmic-ray antiproton measurements and astrophysical implications," The Astrophysical Journal Letters, vol. 247, pp. L105-L109, 1981.

[147] J. Ellis, R. A. Flores, K. Freese, S. Ritz, D. Seckel, and J. Silk, "Cosmic ray constraints on the annihilations of relic particles in the galactic halo," Physics Letters B, vol. 214, no. 3, pp. 403412, 1988.

[148] S. Rudaz and F. W. Stecker, "Cosmic-ray antiprotons, positrons, and gamma rays from halo dark matter annihilation," The Astrophysical Journal, vol. 325, pp. 16-25, 1988.

[149] L. Bergström, J. Edsjö, and P. Ullio, "Cosmic antiprotons as a probe for supersymmetric dark matter?" The Astrophysical Journal, vol. 526, no. 1, 1999.

[150] O. Adriani, G. C. Barbarino, G. A. Bazilevskaya et al., "New measurement of the antiproton-to-proton flux ratio up to 100 $\mathrm{GeV}$ in the cosmic radiation," Physical Review Letters, vol. 102, no. $5,2009$.

[151] O. Adriani, G. C. Barbarino, G. A. Bazilevskaya et al., "PAMELA results on the cosmic-ray antiproton flux from $60 \mathrm{MeV}$ to 180 GeV in kinetic energy," Physical Review Letters, vol. 105, no. 12, 2010.
[152] G. Di Bernardo, C. Evoli, D. Gaggero, D. Grasso, and L. Maccione, "Unified interpretation of cosmic ray nuclei and antiproton recent measurements," Astroparticle Physics, vol. 34, no. 5, pp. 274-283, 2010.

[153] T. Bringmann and P. Salati, "Galactic antiproton spectrum at high energies: Background expectation versus exotic contributions," Physical Review D: Particles, Fields, Gravitation and Cosmology, vol. 75, no. 8, 2007.

[154] F. Donato, D. Maurin, P. Brun, T. Delahaye, and P. Salati, "Constraints on WIMP dark matter from the high energy PAMELA $\bar{p} / p$ Data," Physical Review Letters, vol. 102, no. 7, 2009.

[155] M. Cirelli, D. Gaggero, G. Giesen, M. Taoso, and A. Urbano, "Antiproton constraints on the $\mathrm{GeV}$ gamma-ray excess: a comprehensive analysis," Journal of Cosmology and Astroparticle Physics, vol. 2014, no. 12, 2014.

[156] A. W. Strong, E. Orlando, and T. R. Jaffe, “The interstellar cosmic-ray electron spectrum from synchrotron radiation and direct measurements," Astronomy and Astrophysics, vol. 534, 2011.

[157] G. Di Bernardo, C. Evoli, D. Gaggero, D. Grasso, and L. Maccione, "Cosmic ray electrons, positrons and the synchrotron emission of the Galaxy: consistent analysis and implications," Journal of Cosmology and Astroparticle Physics, vol. 2013, no. 03, 2013.

[158] R. Kappl, A. Reinert, and M. W. Winkler, "AMS-02 antiprotons reloaded," Journal of Cosmology and Astroparticle Physics, vol. 2015, no. 10, 2015.

[159] P. Blasi, "Origin of the positron excess in cosmic rays," Physical Review Letters, vol. 103, no. 5, 2009.

[160] P. Mertsch and S. Sarkar, "AMS-02 data confront acceleration of cosmic ray secondaries in nearby sources," Physical Review D: Particles, Fields, Gravitation and Cosmology, vol. 90, no. 6, 2014.

[161] J. Feng, N. Tomassetti, and A. Oliva, "Bayesian analysis of spatial-dependent cosmic-ray propagation: Astrophysical background of antiprotons and positrons," Physical Review D: Particles, Fields, Gravitation and Cosmology, vol. 94, no. 12, 2016.

[162] Y.-Q. Guo and Q. Yuan, "Understanding the spectral hardenings and radial distribution of Galactic cosmic rays and Fermi diffuse gamma-rays with spatially-dependent propagation," Physical Review D: Particles, Fields, Gravitation and Cosmology, vol. 97, no. 6, 2018.

[163] I. Cholis, D. Hooper, and T. Linden, "Possible evidence for the stochastic acceleration of secondary antiprotons by supernova remnants," Physical Review D, vol. 95, no. 12, 2017.

[164] S.-J. Lin, X.-J. Bi, P.-F. Yin, and Z.-H. Yu, "Implications for dark matter annihilation from the AMS- $02 \bar{p} / p$ ratio," Physical Review D, vol. D95, no. 6, 2017.

[165] X.-J. Huang, C.-C. Wei, Y.-L. Wu, W.-H. Zhang, and Y.-F. Zhou, "Antiprotons from dark matter annihilation through light mediators and a possible excess in AMS-02 $\bar{p} / p$ data," Physical Review D, vol. 95, no. 6, 2017.

[166] J. Feng and H.-H. Zhang, "Dark matter search in space: combined analysis of cosmic ray antiproton-to-proton flux ratio and positron flux measured by AMS-02," The Astrophysical Journal, vol. 858, no. 2, 2018.

[167] S.-J. Lin, X.-J. Bi, J. Feng, P.-F. Yin, and Z.-H. Yu, "Systematic study on the cosmic ray antiproton flux," Physical Review D: Particles, Fields, Gravitation and Cosmology, vol. 96, no. 12, 2017.

[168] A. Cuoco, M. Krämer, and M. Korsmeier, "Novel dark matter constraints from antiprotons in light of AMS-02," Physical Review Letters, vol. 118, no. 19, 2017. 
[169] A. Cuoco, J. Heisig, M. Korsmeier, and M. Krämer, "Probing dark matter annihilation in the Galaxy with antiprotons and gamma rays," Journal of Cosmology and Astroparticle Physics, vol. 2017, no. 10, 2017.

[170] M.-Y. Cui, Q. Yuan, Y.-L. S. Tsai, and Y.-Z. Fan, "Possible dark matter annihilation signal in the AMS-02 antiproton data," Physical Review Letters, vol. 118, no. 19, 2017.

[171] F. Donato, N. Fornengo, and P. Salati, "Antideuterons as a signature of supersymmetric dark matter," Physical Review D: Particles, Fields, Gravitation and Cosmology, vol. 62, no. 4, 2000.

[172] H. Baer and S. Profumo, "Low energy antideuterons: shedding light on dark matter," Journal of Cosmology and Astroparticle Physics, vol. 2005, no. 12, 2005.

[173] R. Duperray, B. Baret, D. Maurin et al., "Flux of light antimatter nuclei near Earth, induced by cosmic rays in the Galaxy and in the atmosphere," Physical Review D: Particles, Fields, Gravitation and Cosmology, vol. 71, no. 8, 2005.

[174] F. Donato, N. Fornengo, and D. Maurin, "Antideuteron fluxes from dark matter annihilation in diffusion models," Physical Review D: Particles, Fields, Gravitation and Cosmology, vol. 78, no. 4, 2008.

[175] E. Carlson, A. Coogan, T. Linden, S. Profumo, A. Ibarra, and S. Wild, "Antihelium from dark matter," Physical Review D: Particles, Fields, Gravitation and Cosmology, vol. 89, no. 7, 2014.

[176] M. Cirelli, N. Fornengo, M. Taoso, and A. Vittino, "Anti-helium from dark matter annihilations," Journal of High Energy Physics, vol. 2014, no. 8, 2014.

[177] H. Fuke et al., "Search for cosmic-ray antideuterons," Physical Review Letters, vol. 95, no. 8, 2005.

[178] K. Abe et al., "Search for antihelium with the BESS-polar spectrometer," Physical Review Letters, vol. 108, no. 13, 2012.

[179] T. Aramaki et al., "Review of the theoretical and experimental status of dark matter identification with cosmic-ray antideuterons," Physics Reports, vol. 618, pp. 1-37, 2016.

[180] A. Coogan and S. Profumo, "Origin of the tentative AMS antihelium events," Physical Review D: Particles, Fields, Gravitation and Cosmology, vol. 96, no. 8, 2017.

[181] P. Chardonnet, J. Orloff, and P. Salati, “The production of antimatter in our galaxy," Physics Letters B, vol. 409, no. 1-4, pp. 313320, 1997.

[182] J. Herms, A. Ibarra, A. Vittino, and S. Wild, "Antideuterons in cosmic rays: sources and discovery potential," Journal of Cosmology and Astroparticle Physics, vol. 2017, no. 02, 2017.

[183] N. Tomassetti and A. Oliva, "Production and acceleration of antinuclei in supernova shockwaves," The Astrophysical Journal Letters, vol. 844, no. 2, 2017.

[184] M. Korsmeier, F. Donato, and N. Fornengo, "Prospects to verify a possible dark matter hint in cosmic antiprotons with antideuterons and antihelium," Physical Review D, vol. 7, no. 10, 2017.

[185] P. Kiraly, J. Szabelski, J. Wdowczyk, and A. W. Wolfendale, "Antiprotons in the cosmic radiation," Nature, vol. 293, pp. 120$122,1981$.

[186] M. S. Turner, "Could primordial black holes be the source of the cosmic ray antiprotons?" Nature, vol. 297, pp. 379-381, 1982.

[187] Q. Yuan, L. Feng, P.-F. Yin et al., Interpretations of the DAMPE electron data, 2017, https://inspirehep.net/record/1639414.

[188] L. J. Gleeson and W. I. Axford, "Solar modulation of galactic cosmic rays," The Astrophysical Journal, vol. 154, p. 1011, 1968.
[189] N. Fornengo, L. Maccione, and A. Vittino, "Dark matter searches with cosmic antideuterons: status and perspectives," Journal of Cosmology and Astroparticle Physics, vol. 2013, no. 09, 2013.

[190] L. Maccione, "Low energy cosmic ray positron fraction explained by charge-sign dependent solar modulation," Physical Review Letters, vol. 110, no. 8, 2013.

[191] A. Vittino, C. Evoli, and D. Gaggero, "Cosmic-ray transport in the heliosphere with HelioProp," in Proceedings of the 35th International Cosmic Ray Conference (ICRC 2017), Bexco, Busan, Korea, 2017.

[192] A. Schwarzschild and C. Zupancic, "Production of tritons, deuterons, nucleons, and mesons by $30-\mathrm{GeV}$ protons on $\mathrm{Al}, \mathrm{Be}$, and Fe targets," Physical Review, vol. 129, no. 2, 1963.

[193] S.-J. Lin, X.-J. Bi, and P.-F. Yin, Expectations of the cosmic antideuteron flux, 2018, https://inspirehep.net/record/1645899.

[194] K. Blum, K. C. Y. Ng, R. Sato, and M. Takimoto, "Cosmic rays, antihelium, and an old navy spotlight," Physical Review D: Particles, Fields, Gravitation and Cosmology, vol. 96, no. 10, 2017.

[195] M. A. Lisa, S. Pratt, R. Soltz, and U. Wiedemann, "Femtoscopy in relativistic heavy ion collisions: two decades of progress," Annual Review of Nuclear and Particle Science, vol. 55, pp. 357402, 2005.

[196] N. J. Shaviv, E. Nakar, and T. Piran, "Inhomogeneity in cosmic ray sources as the origin of the electron spectrum and the PAMELA anomaly," Physical Review Letters, vol. 103, no. 11, 2009.

[197] M. Aguilar et al., "Electron and positron fluxes in primary cosmic rays measured with the alpha magnetic spectrometer on the international space station," Physical Review Letters, vol. 113, no. 12, 2014.

[198] P. D. Serpico, "Possible causes of a rise with energy of the cosmic ray positron fraction," Physical Review D, vol. 79, no. 2, 2009.

[199] D. Grasso, S. Profumo, A. W. Strong et al., "On possible interpretations of the high energy electron-positron spectrum measured by the fermi large area telescope," Astroparticle Physics, vol. 32, no. 2, pp. 140-151, 2009.

[200] P. D. Serpico, "Astrophysical models for the origin of the positron "excess"," Astroparticle Physics, vol. 39-40, pp. 2-11, 2012.

[201] X.-G. He, "Dark matter annihilation explanation for $\mathrm{e}^{ \pm}$excesses in cosmic ray," Modern Physics Letters A, vol. 24, no. 27, pp. 21392160, 2009

[202] T. R. Slatyer, N. Padmanabhan, and D. P. Finkbeiner, "CMB constraints on WIMP annihilation: Energy absorption during the recombination epoch," Physical Review D: Particles, Fields, Gravitation and Cosmology, vol. 80, no. 4, 2009.

[203] S. Galli, F. Iocco, G. Bertone, and A. Melchiorri, "CMB constraints on dark matter models with large annihilation cross section," Physical Review D: Particles, Fields, Gravitation and Cosmology, vol. 80, no. 2, 2009.

[204] J. Buch, P. Ralegankar, and V. Rentala, "Late decaying 2component dark matter scenario as an explanation of the AMS02 positron excess," Journal of Cosmology and Astroparticle Physics, vol. 2017, no. 10, 2017.

[205] M. Ibe, H. Murayama, and T. T. Yanagida, "Breit-Wigner enhancement of dark matter annihilation," Physical Review D: Particles, Fields, Gravitation and Cosmology, vol. 79, no. 9, 2009.

[206] W.-L. Guo and Y.-L. Wu, "Enhancement of dark matter annihilation via Breit-Wigner resonance," Physical Review D: Particles, Fields, Gravitation and Cosmology, vol. 79, no. 5, 2009. 
[207] Y. Bai, J. Berger, and S. Lu, "Supersymmetric resonant dark matter: a thermal model for the AMS-02 positron excess," Physical Review D: Particles, Fields, Gravitation and Cosmology, vol. 97, no. 11, 2018.

[208] Q.-F. Xiang, X.-J. Bi, S.-J. Lin, and P.-F. Yin, "A dark matter model that reconciles tensions between the cosmic-ray $\mathrm{e}^{ \pm}$excess and the gamma-ray and CMB constraints," Physics Letters B, vol. 773, pp. 448-454, 2017.

[209] M. Ackermann et al., "Searches for cosmic-ray electron anisotropies with the fermi large area telescope," Physical Review D: Particles, Fields, Gravitation and Cosmology, vol. 82, no. 9, 2010.

[210] S. Abdollahi et al., "Search for cosmic-ray electron and positron anisotropies with seven years of fermi large area telescope data," Physical Review Letters, vol. 118, no. 9, 2017.

[211] G. Di Bernardo, C. Evoli, D. Gaggero, D. Grasso, L. Maccione, and M. N. Mazziotta, "Implications of the cosmic ray electron spectrum and anisotropy measured with Fermi-LAT," Astroparticle Physics, vol. 34, no. 7, pp. 528-538, 2011.

[212] J. C. Joshi and S. Razzaque, "A self-consistent model of cosmicray fluxes and positron excess: Roles of nearby pulsars and a sub-dominant source population," Journal of Cosmology and Astroparticle Physics, vol. 2017, no. 9, 2017.

[213] S. Manconi, M. Di Mauro, and F. Donato, "Dipole anisotropy in cosmic electrons and positrons: inspection on local sources," Journal of Cosmology and Astroparticle Physics, vol. 2017, no. 01, 2017.

[214] P. Mertsch, "Cosmic ray electrons and positrons from discrete stochastic sources," Journal of Cosmology and Astroparticle Physics, vol. 2011, no. 02, 2011.

[215] A. U. Abeysekara et al., "Extended gamma-ray sources around pulsars constrain the origin of the positron flux at Earth," Science, vol. 358, pp. 911-914, 2017.

[216] R. López-Coto and G. Giacinti, "Constraining the properties of the magnetic turbulence in the Geminga region using HAWC $\gamma$-ray data," Monthly Notices of the Royal Astronomical Society, vol. 479, pp. 4526-4534, 2018.

[217] P. Lipari, "Interpretation of the cosmic ray positron and antiproton fluxes," Physical Review D, vol. 95, no. 6, 2017.

[218] F. Aharonian et al., "Energy spectrum of cosmic-ray electrons at TeV energies," Physical Review Letters, vol. 101, no. 26, 2008.

[219] DAMPE Collaboration, "Direct detection of a break in the teraelectronvolt cosmic-ray spectrum of electrons and positrons," Nature, vol. 552, pp. 63-66, 2017.

[220] O. Adriani et al., "Energy spectrum of cosmic-ray electron and positron from $10 \mathrm{GeV}$ to $3 \mathrm{TeV}$ observed with the calorimetric electron telescope on the international space station," Physical Review Letters, vol. 119, no. 18, 2017.

[221] M. Ackermann et al., "Fermi-LAT observations of the diffuse gamma-ray emission: implications for cosmic rays and the interstellar medium," The Astrophysical Journal, vol. 750, no. 1, 2012.

[222] I. Cholis, M. Tavakoli, C. Evoli, L. Maccione, and P. Ullio, "Diffuse galactic gamma rays at intermediate and high latitudes. I. Constraints on the ISM properties," Journal of Cosmology and Astroparticle Physics, vol. 2012, no. 05, 2012.

[223] M. Pohl, P. Englmaier, and N. Bissantz, "Three-dimensional distribution of molecular gas in the barred milky way," The Astrophysical Journal, vol. 677, no. 1, 2008.

[224] M. Tavakoli, Three dimensional distribution of atomic hydrogen in the milky way, 2012, https://arxiv.org/abs/1207.6150.
[225] T. A. Porter, I. V. Moskalenko, and A. W. Strong, "Inverse compton emission from galactic supernova remnants: effect of the interstellar radiation field," The Astrophysical Journal Letters, vol. 648, no. 1, 2006.

[226] M. Tavakoli, I. Cholis, C. Evoli, and P. Ullio, "Constraints on dark matter annihilations from diffuse gamma-ray emission in the Galaxy," Journal of Cosmology and Astroparticle Physics, vol. 2014, no. 01, 2014.

[227] A. D. Erlykin and A. W. Wolfendale, "Cosmic rays in the inner galaxy and the diffusion properties of the interstellar medium," Astroparticle Physics, vol. 42, pp. 70-75, 2013.

[228] D. Gaggero, D. Grasso, A. Marinelli, A. Urbano, and M. Valli, "The gamma-ray and neutrino sky: a consistent picture of fermi-lat, milagro, and icecube results," The Astrophysical Journal Letters, vol. 815, no. 2, 2015.

[229] F. Acero et al., "Development of the model of galactic interstellar emission for standard point-source analysis of fermi large area telescope data," The Astrophysical Journal Supplement Series, vol. 223, no. 2, 2016.

[230] R. Yang, F. Aharonian, and C. Evoli, "Radial distribution of the diffuse $\gamma$-ray emissivity in the Galactic disk," Physical Review D: Particles, Fields, Gravitation and Cosmology, vol. 93, no. 12, 2016.

[231] E. Nezri, J. Lavalle, and R. Teyssier, "Indirect dark matter searches: Towards a consistent top-bottom approach for studying the gamma-ray signals and associated backgrounds," Physical Review D: Particles, Fields, Gravitation and Cosmology, vol. 86, no. 6, 2012.

[232] E. Charles et al., "Sensitivity projections for dark matter searches with the fermi large area telescope," Physics Reports, vol. 636, pp. $1-46,2016$.

[233] J. Conrad and O. Reimer, "Indirect dark matter searches in gamma- and cosmic rays," Nature Physics, vol. 13, pp. 224-231, 2017.

[234] V. Springel, S. D. M. White, C. S. Frenk et al., "Prospects for detecting supersymmetric dark matter in the Galactic halo," Nature, vol. 456, no. 4, pp. 73-76, 2008.

[235] M. Schaller et al., "Dark matter annihilation radiation in hydrodynamic simulations of Milky Way haloes," Monthly Notices of the Royal Astronomical Society, vol. 455, no. 4, pp. 4442-4451, 2016.

[236] M. Taoso, G. Bertone, and A. Masiero, "Dark matter candidates: a ten-point test," Journal of Cosmology and Astroparticle Physics, vol. 2008, no. 03, 2008.

[237] L. Bergstrom and P. Ullio, "Full one-loop calculation of neutralino annihilation into two photons," Nuclear Physics B, vol. 504, no. 1-2, pp. 27-44, 1997.

[238] P. Ullio and L. Bergström, "Neutralino annihilation into a photon and a Z boson," Physical Review D: Particles, Fields, Gravitation and Cosmology, vol. 57, no. 3, 1998.

[239] T. Bringmann, X. Huang, A. Ibarra, S. Vogl, and C. Weniger, "Fermi LAT search for internal bremsstrahlung signatures from dark matter annihilation," Journal of Cosmology and Astroparticle Physics, vol. 2012, no. 07, 2012.

[240] C. Weniger, "A tentative gamma-ray line from dark matter annihilation at the fermi large area telescope," Journal of Cosmology and Astroparticle Physics, vol. 2012, no. 8, 2012.

[241] D. P. Finkbeiner, M. Su, and C. Weniger, "Is the $130 \mathrm{GeV}$ line real? A search for systematics in the Fermi-LAT data," Journal of Cosmology and Astroparticle Physics, vol. 2013, no. 01, 2013.

[242] C. Weniger, M. Su, D. P. Finkbeiner, T. Bringmann, and N. Mirabal, Closing in on the fermi line with a new observation strategy, 2013, https://arxiv.org/abs/1305.4710. 
[243] M. Ackermann et al., "Updated search for spectral lines from galactic dark matter interactions with pass 8 data from the fermi large area telescope," Physical Review D, vol. 91, no. 12, 2015.

[244] H. Abdalla et al., "H.E.S.S. limits on line-like dark matter signatures in the $100 \mathrm{GeV}$ to $2 \mathrm{TeV}$ energy range close to the Galactic Centre," Physical Review Letters, vol. 117, no. 15, 2016.

[245] D. Gaggero, M. Taoso, A. Urbano, M. Valli, and P. Ullio, "Towards a realistic astrophysical interpretation of the gammaray Galactic center excess," Journal of Cosmology and Astroparticle Physics, vol. 2015, no. 12, 2015.

[246] V. Vitale, A. Morselli, and for the Fermi/LAT Collaboration, Indirect search for dark matter from the center of the milky way with the fermi-large area telescope, 2009, https://arxiv .org/abs/0912.3828.

[247] L. Goodenough and D. Hooper, Possible evidence for dark matter annihilation in the inner milky way from the fermi gamma ray space telescope, 2009, https://arxiv.org/abs/0910.2998.

[248] D. Hooper and L. Goodenough, "Dark matter annihilation in the galactic center as seen by the fermi gamma ray space telescope," Physics Letters B, vol. 697, no. 5, pp. 412-428, 2011.

[249] A. Boyarsky, D. Malyshev, and O. Ruchayskiy, "A comment on the emission from the Galactic Center as seen by the Fermi telescope," Physics Letters B, vol. 705, no. 3, pp. 165-169, 2011.

[250] D. Hooper and T. Linden, "On the origin of the gamma rays from the galactic center," Physical Review D, vol. 84, no. 12, 2011.

[251] K. N. Abazajian and M. Kaplinghat, "Detection of a gamma-ray source in the galactic center consistent with extended emission from dark matter annihilation and concentrated astrophysical emission," Physical Review D, vol. 86, no. 8, 2012, Erratum: vol. 87, no. 12, 2013.

[252] D. Hooper and T. R. Slatyer, "Two emission mechanisms in the fermi bubbles: a possible signal of annihilating dark matter," Physics of the Dark Universe, vol. 2, no. 3, pp. 118-138, 2013.

[253] C. Gordon and O. Macías, "Dark matter and pulsar model constraints from Galactic Center Fermi-LAT gamma-ray observations," Physical Review D: Particles, Fields, Gravitation and Cosmology, vol. 88, no. 8, 2013, Erratum: vol. 89, no. 4, 2014.

[254] W.-C. Huang, A. Urbano, and W. Xue, Fermi bubbles under dark matter scrutiny. Part I: astrophysical analysis, 2013, https://arxiv.org/abs/1307.6862.

[255] O. Macias and C. Gordon, "Contribution of cosmic rays interacting with molecular clouds to the galactic center gammaray excess," Physical Review D, vol. 89, no. 6, 2014.

[256] K. N. Abazajian, N. Canac, S. Horiuchi, and M. Kaplinghat, "Astrophysical and dark matter interpretations of extended gamma-ray emission from the Galactic Center," Physical Review D: Particles, Fields, Gravitation and Cosmology, vol. 90, no. 2, 2014.

[257] T. Daylan, D. P. Finkbeiner, D. Hooper et al., "The characterization of the gamma-ray signal from the central Milky Way: A case for annihilating dark matter," Physics of the Dark Universe, vol. 12, pp. 1-23, 2016.

[258] B. Zhou, Y.-F. Liang, X. Huang et al., "GeV excess in the Milky Way: The role of diffuse galactic gamma-ray emission templates," Physical Review D: Particles, Fields, Gravitation and Cosmology, vol. 91, no. 12, 2015.

[259] F. Calore, I. Cholis, and C. Weniger, "Background model systematics for the Fermi GeV excess," Journal of Cosmology and Astroparticle Physics, vol. 2015, no. 03, 2015.

[260] E. Carlson, S. Profumo, and T. Linden, "Cosmic-ray injection from star-forming regions," Physical Review Letters, vol. 117, no. 11, 2016.
[261] S. Horiuchi, M. Kaplinghat, and A. Kwa, "Investigating the uniformity of the excess gamma rays towards the galactic center region," Journal of Cosmology and Astroparticle Physics, vol. 2016, no. 11, 2016.

[262] M. Ackermann et al., "The fermi galactic center gev excess and implications for dark matter," The Astrophysical Journal, vol. 840, no. 1, 2017.

[263] W. de Boer, L. Bosse, I. Gebauer, A. Neumann, and P. L. Biermann, "Molecular clouds as origin of the Fermi gamma-ray GeV excess," Physical Review D: Particles, Fields, Gravitation and Cosmology, vol. 96, no. 4, 2017.

[264] M. Su, T. R. Slatyer, and D. P. Finkbeiner, "Giant gamma-ray bubbles from fermi-LAT: Active galactic nucleus activity or bipolar galactic wind?" The Astrophysical Journal, vol. 724, no. 2, 2010.

[265] W.-C. Huang, A. Urbano, and W. Xue, "Fermi bubbles under dark matter scrutiny part II: Particle physics analysis," Journal of Cosmology and Astroparticle Physics, vol. 2014, no. 04, 2014.

[266] A. Alves, S. Profumo, F. S. Queiroz, and W. Shepherd, "Effective field theory approach to the Galactic Center gamma-ray excess," Physical Review D: Particles, Fields, Gravitation and Cosmology, vol. 90, no. 11, 2014.

[267] A. Berlin, D. Hooper, and S. D. McDermott, "Simplified dark matter models for the Galactic Center gamma-ray excess," Physical Review D: Particles, Fields, Gravitation and Cosmology, vol. 89, no. 11, 2014.

[268] M. Abdullah, A. DiFranzo, A. Rajaraman, T. M. Tait, P. Tanedo, and A. M. Wijangco, "Hidden on-shell mediators for the galactic center $\gamma$-ray excess," Physical Review D: Particles, Fields, Gravitation and Cosmology, vol. 90, no. 3, 2014.

[269] P. Agrawal, B. Batell, P. J. Fox, and R. Harnik, "WIMPs at the galactic center," Journal of Cosmology and Astroparticle Physics, vol. 2015, no. 05, 2015.

[270] F. Calore, I. Cholis, C. McCabe, and C. Weniger, "A tale of tails: Dark matter interpretations of the Fermi $\mathrm{GeV}$ excess in light of background model systematics," Physical Review D: Particles, Fields, Gravitation and Cosmology, vol. 91, no. 6, 2015.

[271] M. Kaplinghat, T. Linden, and H.-B. Yu, "Galactic center excess in gamma rays from annihilation of self-interacting dark matter," Physical Review Letters, vol. 114, no. 21, 2015.

[272] C. Karwin, S. Murgia, T. M. P. Tait, T. A. Porter, and P. Tanedo, "Dark matter interpretation of the Fermi-LAT observation toward the galactic center," Physical Review D: Particles, Fields, Gravitation and Cosmology, vol. 95, no. 10, 2017.

[273] J. Petrović, P. D. Serpico, and G. Zaharijas, "Millisecond pulsars and the Galactic Center gamma-ray excess: the importance of luminosity function and secondary emission," Journal of Cosmology and Astroparticle Physics, vol. 2015, no. 02, 2015.

[274] K. N. Abazajian, "The Consistency of Fermi-LAT observations of the galactic center with a millisecond pulsar population in the central stellar cluster," Journal of Cosmology and Astroparticle Physics, vol. 2011, no. 3, 2011.

[275] Q. Yuan and B. Zhang, "Millisecond pulsar interpretation of the Galactic center gamma-ray excess," Journal of High Energy Astrophysics, vol. 3-4, pp. 1-8, 2014.

[276] D. Hooper, I. Cholis, T. Linden, J. M. Siegal-Gaskins, and T. R. Slatyer, "Millisecond pulsars cannot account for the inner Galaxy's GeV excess," Physical Review D: Particles, Fields, Gravitation and Cosmology, vol. 88, no. 8, 2013. 
[277] I. Cholis, D. Hooper, and T. Linden, "Challenges in explaining the Galactic Center gamma-ray excess with millisecond pulsars," Journal of Cosmology and Astroparticle Physics, vol. 2015, no. 06, 2015.

[278] E. Carlson and S. Profumo, "Cosmic ray protons in the inner galaxy and the galactic center gamma-ray excess," Physical Review D, vol. 90, no. 2, 2014.

[279] J. Petrovi, P. D. Serpico, and G. Zaharija, "Galactic Center gamma-ray "excess" from an active past of the galactic centre?" Journal of Cosmology and Astroparticle Physics, vol. 2014, no. 10, 2014.

[280] I. Cholis, C. Evoli, F. Calore, T. Linden, C. Weniger, and D. Hooper, "The galactic center $\mathrm{GeV}$ excess from a series of leptonic cosmic-ray outbursts," Journal of Cosmology and Astroparticle Physics, vol. 2015, no. 12, 2015.

[281] M. Selig, V. Vacca, N. Oppermann, and T. A. Enßlin, “The denoised, deconvolved, and decomposed fermi $\gamma$-ray sky - An application of the $\mathrm{D}^{3} \mathrm{PO}$ algorithm," Astronomy and Astrophysics, vol. 581, 2015.

[282] D. F. Figer, R. M. Rich, S. S. Kim, M. Morris, and E. Serabyn, "An Extended Star Formation History for the Galactic Center from hubble space telescope NICMOS observations," The Astrophysical Journal, vol. 601, no. 1, 2004.

[283] R. M. Crocker, D. I. Jones, F. Aharonian et al., "Wild at heart: the particle astrophysics of the galactic centre," Monthly Notices of the Royal Astronomical Society, vol. 413, no. 2, pp. 763-788, 2011.

[284] K. Ferriere, W. Gillard, and P. Jean, "Spatial distribution of interstellar gas in the innermost $3 \mathrm{kpc}$ of our galaxy," Astronomy and Astrophysics, vol. 467, pp. 611-627, 2007.

[285] D. F. Figer et al., "Massive stars in the arches cluster," The Astrophysical Journal, vol. 581, no. 1, 2002.

[286] T. Montmerle, "On gamma-ray sources, supernova remnants, OB associations, and the origin of cosmic rays," Astrophysical Journal, vol. 231, pp. 95-110, 1979.

[287] E. Carlson, T. Linden, and S. Profumo, "Improved cosmic-ray injection models and the Galactic Center gamma-ray excess," Physical Review D: Particles, Fields, Gravitation and Cosmology, vol. 94, no. 6, 2016.

[288] M. Schmidt, "The rate of star formation," Astrophysical Journal, vol. 129, p. 243, 1959.

[289] M. Ajello et al., "Fermi-LAT observations of high-energy $\gamma$-ray emission toward the galactic center," The Astrophysical Journal, vol. 819, no. 1, 2016.

[290] R. Bartels, S. Krishnamurthy, and C. Weniger, "Strong support for the millisecond pulsar origin of the galactic center $\mathrm{GeV}$ excess," Physical Review Letters, vol. 116, no. 5, 2016.

[291] S. K. Lee, M. Lisanti, B. R. Safdi, T. R. Slatyer, and W. Xue, "Evidence for unresolved $\gamma$-ray point sources in the inner galaxy," Physical Review Letters, vol. 116, no. 5, 2016.

[292] D. Hooper and G. Mohlabeng, "The gamma-ray luminosity function of millisecond pulsars and implications for the $\mathrm{GeV}$ excess," Journal of Cosmology and Astroparticle Physics, vol. 2016, no. 03, 2016.

[293] H. Ploeg, C. Gordon, R. Crocker, and O. Macias, "Consistency between the luminosity function of resolved millisecond pulsars and the galactic center excess," Journal of Cosmology and Astroparticle Physics, vol. 2017, no. 08, 2017.

[294] M. Ajello et al., Characterizing the population of pulsars in the inner Galaxy with the Fermi Large Area Telescope, 2017, https://arxiv.org/abs/1711.04778.
[295] E. Storm, C. Weniger, and F. Calore, "SkyFACT: highdimensional modeling of gamma-ray emission with adaptive templates and penalized likelihoods," Journal of Cosmology and Astroparticle Physics, vol. 2017, no. 08, 2017.

[296] R. Bartels, E. Storm, C. Weniger, and F. Calore, The FermiLAT GeV excess traces stellar mass in the galactic bulge, 2017, https://arxiv.org/abs/1705.00009.

[297] F. Calore, M. Di Mauro, F. Donato, J. W. Hessels, and C. Weniger, "Radio detection prospects for a bulge population of millisecond pulsars as suggested by Fermi-LAT observations of the inner galaxy," The Astrophysical Journal, vol. 827, no. 2, 2016.

[298] M. Ackermann et al., "Observations of M31 and M33 with the fermi large area telescope: a galactic center excess in andromeda?" The Astrophysical Journal, vol. 836, no. 2, 2017.

[299] C. Eckner, X. Hou, P. D. Serpico et al., "Millisecond pulsar origin of the Galactic center excess and extended gamma-ray emission from Andromeda - a closer look," The Astrophysical Journal, vol. 862, no. 1, 79 pages, 2018.

[300] G. Lake, "Detectability of $\gamma$-rays from clumps of dark matter," Nature, vol. 346, pp. 39-40, 1990.

[301] N. W. Evans, F. Ferrer, and S. Sarkar, "A travel guide to the dark matter annihilation signal," Physical Review D: Particles, Fields, Gravitation and Cosmology, vol. 69, no. 12, 2004.

[302] L. E. Strigari, S. M. Koushiappas, J. S. Bullock, and M. Kaplinghat, "Precise constraints on the dark matter content of Milky Way dwarf galaxies for gamma-ray experiments," Physical Review D: Particles, Fields, Gravitation and Cosmology, vol. 75, no. 8, 2007.

[303] L. E Strigari, S. M. Koushiappas, J. S. Bullock et al., "The most dark-matter-dominated galaxies: predicted gamma-ray signals from the faintest milky way dwarfs," The Astrophysical Journal, vol. 678 , no. 2, 2008.

[304] M. Ackermann et al., "Constraining dark matter models from a combined analysis of milky way satellites with the fermi large area telescope," Physical Review Letters, vol. 107, no. 24, 2011.

[305] M. Ackermann et al., "Searching for dark matter annihilation from milky way dwarf spheroidal galaxies with six years of fermi large area telescope data," Physical Review Letters, vol. 115, no. 23, 2015.

[306] M. L. Ahnen et al., "Limits to dark matter annihilation crosssection from a combined analysis of MAGIC and Fermi-LAT observations of dwarf satellite galaxies," Journal of Cosmology and Astroparticle Physics, vol. 2016, no. 02, 2016.

[307] A. Albert et al., "Searching for dark matter annihilation in recently discovered milky way satellites with Fermi-LAT," The Astrophysical Journal, vol. 834, no. 2, 2017.

[308] S. Archambault et al., "Dark matter constraints from a joint analysis of dwarf spheroidal galaxy observations with VERITAS," Physical Review D: Particles, Fields, Gravitation and Cosmology, vol. 95, no. 8, 2017.

[309] K. N. Abazajian and R. E. Keeley, "Bright gamma-ray Galactic Center excess and dark dwarfs: Strong tension for dark matter annihilation despite Milky Way halo profile and diffuse emission uncertainties," Physical Review D, vol. 93, no. 8, 2016.

[310] R. Keeley, K. Abazajian, A. Kwa, N. Rodd, and B. Safdi, "What the Milky Way's Dwarfs tell us about the Galactic Center extended gamma-ray excess," Physical Review D, vol. 97, no. 10, 2018.

[311] V. Bonnivard, C. Combet, D. Maurin, and M. G. Walker, "Spherical Jeans analysis for dark matter indirect detection in dwarf spheroidal galaxies - impact of physical parameters and 
triaxiality," Monthly Notices of the Royal Astronomical Society, vol. 446, no. 3, pp. 3002-3021, 2015.

[312] P. Ullio and M. Valli, "A critical reassessment of particle Dark Matter limits from dwarf satellites," Journal of Cosmology and Astroparticle Physics, vol. 2016, no. 07, 2016.

[313] K. Hayashi, K. Ichikawa, S. Matsumoto, M. Ibe, M. N. Ishigaki, and H. Sugai, "Dark matter annihilation and decay from nonspherical dark halos in galactic dwarf satellites," Monthly Notices of the Royal Astronomical Society, vol. 461, no. 3, pp. 2914-2928, 2016.

[314] K. Ichikawa, M. N. Ishigaki, S. Matsumoto et al., "Foreground effect on the J-factor estimation of classical dwarf spheroidal galaxies," Monthly Notices of the Royal Astronomical Society, vol. 468, no. 3, pp. 2884-2896, 2017.

[315] A. Geringer-Sameth, M. G. Walker, S. M. Koushiappas et al., "Indication of gamma-ray emission from the newly discovered dwarf galaxy reticulum II," Physical Review Letters, vol. 115, no. 8, 2015.

[316] D. Hooper and T. Linden, "On the gamma-ray emission from reticulum II and other dwarf galaxies," Journal of Cosmology and Astroparticle Physics, vol. 2015, no. 09, 2015.

[317] V. Bonnivard, C. Combet, D. Maurin et al., "Dark matter annihilation and decay profiles for the reticulum ii dwarf spheroidal galaxy," The Astrophysical Journal Letters, vol. 808, no. 2, 2015.

[318] M. Regis, L. Richter, and S. Colafrancesco, "Dark matter in the Reticulum II dSph: a radio search," Journal of Cosmology and Astroparticle Physics, vol. 2017, no. 07, 2017.

[319] S. Colafrancesco, S. Profumo, and P. Ullio, "Detecting dark matter WIMPs in the Draco dwarf: A multiwavelength perspective," Physical Review D: Particles, Fields, Gravitation and Cosmology, vol. 75 , no. 2, 2007.

[320] S. Profumo and P. Ullio, Multi-wavelength searches for particle dark matter, 2010, https://arxiv.org/abs/1001.4086.

[321] M. Regis, S. Colafrancesco, S. Profumo, W. J. G. de Blok, M. Massardi, and L. Richter, "Local Group dSph radio survey with ATCA (III): constraints on particle dark matter," Journal of Cosmology and Astroparticle Physics, vol. 2014, no. 10, 2014.

[322] A. De Angelis, V. Tatischeff, I. A. Grenier et al., Science with e-ASTROGAM (A space mission for MeV-GeV gamma-ray astrophysics), 2017, https://arxiv.org/abs/1711.01265.

[323] A. A. Moiseev et al., Compton-pair production space telescope (ComPair) for MeV gamma-ray astronomy, 2015, https://arxiv .org/abs/1508.07349.

[324] S. D. Hunter et al., "A Pair Production Telescope for MediumEnergy Gamma-Ray Polarimetry," Astroparticle Physics, vol. 59, pp. 18-28, 2014.

[325] R. Essig, E. Kuflik, S. D. McDermott, T. Volansky, and K. M. Zurek, "Constraining light dark matter with diffuse X-ray and gamma-ray observations," Journal of High Energy Physics, vol. 2013, no. 11, 2013.

[326] K. K. Boddy and J. Kumar, "Indirect detection of dark matter using mev-range gamma-ray telescopes," Physical Review D, vol. 92, no. 2, 2015.

[327] A. W. Strong, H. Bloemen, R. Diehl, W. Hermsen, and V. Schoenfelder, "COMPTEL skymapping: a new approach using parallel computing," Astrophysical Letters and Communications, vol. 39, p. 209, 1999.

[328] S. D. Hunter et al., "EGRET observations of the diffuse gammaray emission from the galactic plane," The Astrophysical Journal, vol. 481, no. 1, 1997.
[329] R. Bartels, D. Gaggero, and C. Weniger, "Prospects for indirect dark matter searches with $\mathrm{MeV}$ photons," Journal of Cosmology and Astroparticle Physics, vol. 2017, no. 05, 2017.

[330] C. Bœhm and Y. Ascasibar, "More evidence in favor of light dark matter particles?" Physical Review D: Particles, Fields, Gravitation and Cosmology, vol. 70, no. 11, 2004.

[331] C. Boehm, D. Hooper, J. Silk, M. Casse, and J. Paul, "MeV Dark Matter: Has It Been Detected?” Physical Review Letters, vol. 92, no. 10, 2004.

[332] E. Lorenz and The MAGIC Collaboration, "Status of the $17 \mathrm{~m}$ $\varnothing$ MAGIC telescope," New Astronomy Reviews, vol. 48, no. 5-6, pp. 339-344, 2004.

[333] A. U. Abeysekara, R. Alfaro, C. Alvarez et al., "The sensitivity of HAWC to high-mass dark matter annihilations," Physical Review D, vol. 90, no. 12, 2014.

[334] A. Abramowski, F. Acero, F. Aharonian et al., "Search for a dark matter annihilation signal from the galactic center halo with H.E.S.S," Physical Review Letters, vol. 106, no. 16, 2011.

[335] M. Doro, J. Conrad, D. Emmanoulopoulos et al., "Dark matter and fundamental physics with the cherenkov telescope array," Astroparticle Physics, vol. 43, pp. 189-214, 2013.

[336] H. Silverwood, C. Weniger, P. Scott, and G. Bertone, "A realistic assessment of the CTA sensitivity to dark matter annihilation," Journal of Cosmology and Astroparticle Physics, vol. 2015, no. 03, 2015.

[337] J. Conrad, "CTA in the Context of Searches for Particle Dark Matter - a glimpse," in Proceedings of the 6th International Symposium on High Energy Gamma-Ray Astronomy, vol. 1792 of American Institute of Physics Conference Series, 2017.

[338] A. Morselli, The dark matter programme of the cherenkov telescope array, 2017, https://arxiv.org/abs/1709.01483.

[339] F. Aharonian, A. G. Akhperjanian, A. R. Bazer-Bachi et al., "Discovery of very-high-energy gamma-rays from the galactic centre ridge," Nature, vol. 439, pp. 695-698, 2006.

[340] H.E.S.S. Collaboration, "Acceleration of petaelectronvolt protons in the galactic centre," Nature, vol. 531, pp. 476-479, 2016.

[341] H.E.S.S. Collaboration, "Characterising the VHE diffuse emission in the central 200 parsecs of our Galaxy with H.E.S.S," Astronomy and Astrophysics, vol. 612, 2018.

[342] M. Hatten, C. Combet, G. Maier, and D. Maurin, "Dark matter substructure modelling and sensitivity of the cherenkov telescope array to galactic dark halos," Journal of Cosmology and Astroparticle Physics, vol. 2016, no. 09, 2016.

[343] V. Lefranc, G. A. Mamon, and P. Panci, "Prospects for annihilating Dark Matter towards Milky Way's dwarf galaxies by the Cherenkov Telescope Array," Journal of Cosmology and Astroparticle Physics, vol. 2016, no. 09, 2016.

[344] V. Lefranc, E. Moulin, P. Panci, F. Sala, and J. Silk, "Dark Matter in $\gamma$ lines: Galactic Center vs. dwarf galaxies," Journal of Cosmology and Astroparticle Physics, vol. 2016, no. 09, 2016.

[345] J. Chang, G. Ambrosi, Q. An et al., "The dark matter particle explorer mission," Astroparticle Physics, vol. 95, pp. 6-24, 2017.

[346] P. S. Marrocchesi, "CALET: a high energy astroparticle physics experiment on the ISS," in Proceedings of the MG14 Meeting on General Relativity, 2015.

[347] E. S. Seo, T. Anderson, D. Angelaszek et al., "Cosmic ray energetics and mass for the international space station (ISSCREAM)," Advances in Space Research, vol. 53, no. 10, pp. 14511455, 2014.

[348] R. A. Ong, T. Aramaki, R. Bird et al., The GAPS experiment to search for dark matter using low-energy antimatter, 2017, https://arxiv.org/abs/1710.00452. 
[349] V. S. Ptuskin, I. V. Moskalenko, F. C. Jones, A. W. Strong, and V. N. Zirakashvili, "Dissipation of magnetohydrodynamic waves on energetic particles: Impact on interstellar turbulence and cosmic-ray transport," The Astrophysical Journal, vol. 642, no. 2, pp. 902-916, 2006.

[350] R. P. Duperray, C.-Y. Huang, K. V. Protasov, and M. Buénerd, "Parameterization of the antiproton inclusive production cross section on nuclei," Physical Review D, vol. 68, no. 9, 2003.

[351] M. di Mauro, F. Donato, A. Goudelis, and P. D. Serpico, "New evaluation of the antiproton production cross section for cosmic ray studies," Physical Review D: Particles, Fields, Gravitation and Cosmology, vol. 90, no. 8, 2014.

[352] R. Kappl and M. W. Winkler, "The cosmic ray antiproton background for AMS-02," Journal of Cosmology and Astroparticle Physics, vol. 2014, no. 9, 2014.

[353] M. W. Winkler, "Cosmic ray antiprotons at high energies," Journal of Cosmology and Astroparticle Physics, vol. 2017, no. 2, 2017.

[354] T. Pierog, I. Karpenko, J. M. Katzy, E. Yatsenko, and K. Werner, "EPOS LHC: Test of collective hadronization with data measured at the CERN Large Hadron Collider," Physical Review C: Nuclear Physics, vol. 92, no. 3, 2015.

[355] R. Engel, T. K. Gaisser, T. Stanev, and P. Lipari, "Air shower calculations with the new version of SIBYLL," in Proceedings of the 26th International Cosmic Ray Conference: Invited, Rapporteur, and Highlight Papers, vol. 1, pp. 415-418, Salt Lake City, Utah, UT, USA, August 17-25, 1999.

[356] S. Ostapchenko, "Monte Carlo treatment of hadronic interactions in enhanced Pomeron scheme: QGSJET-II model," Physical Review D: Particles, Fields, Gravitation, and Cosmology, vol. 83, no. 1, 2011. 

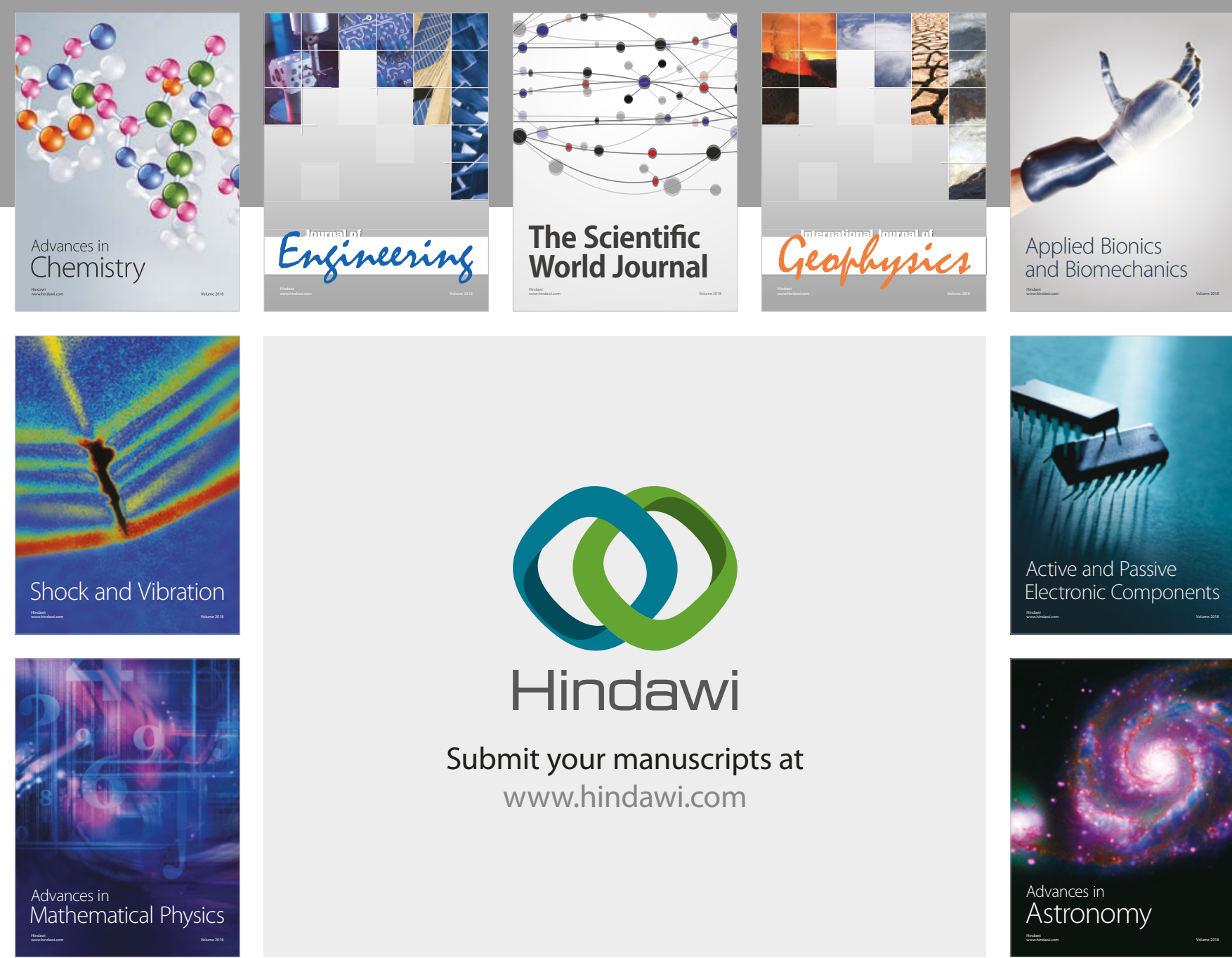

Submit your manuscripts at

www.hindawi.com

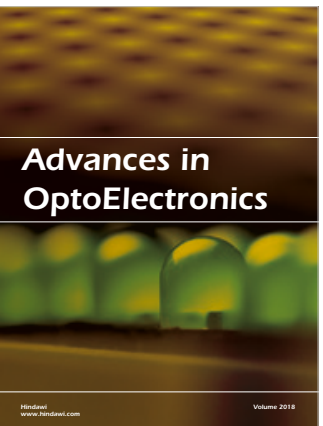

\section{Rotcting Machinery}
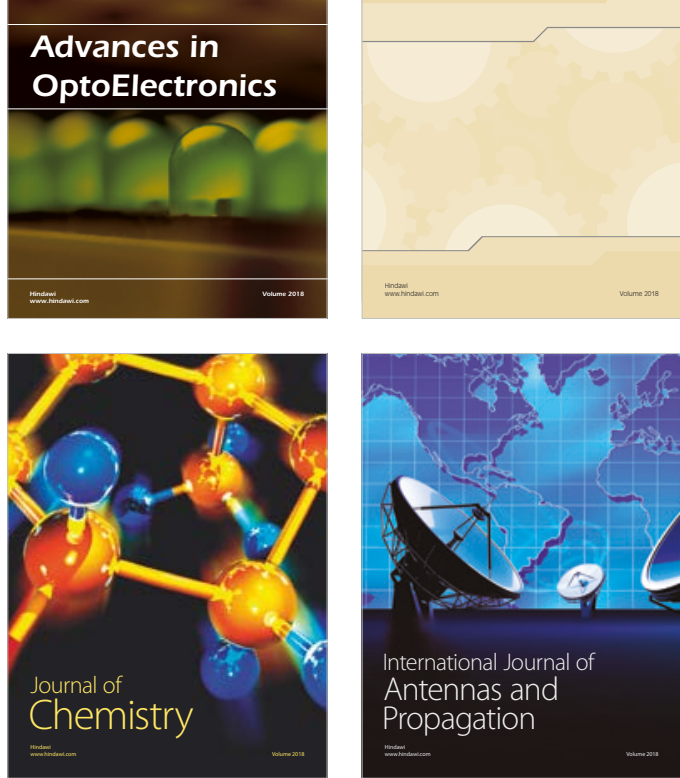

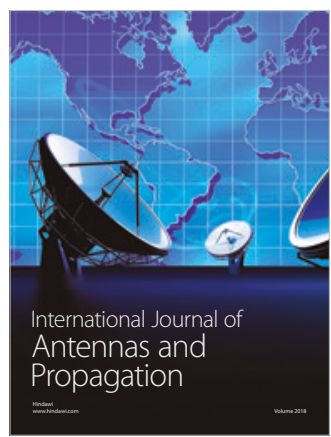

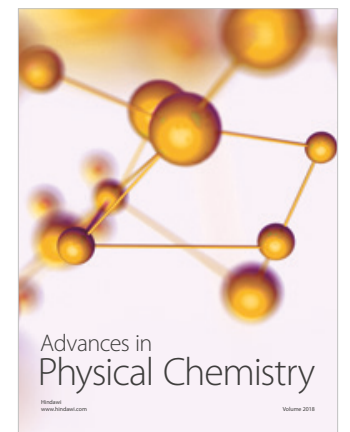

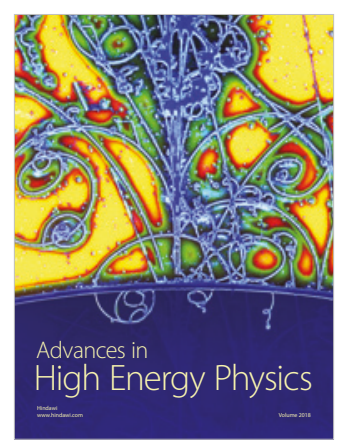

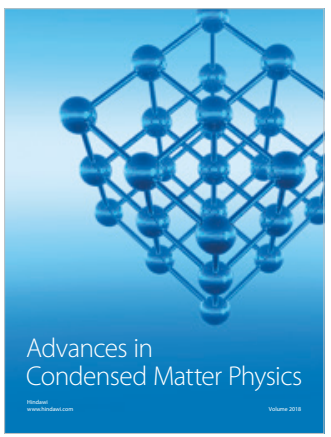

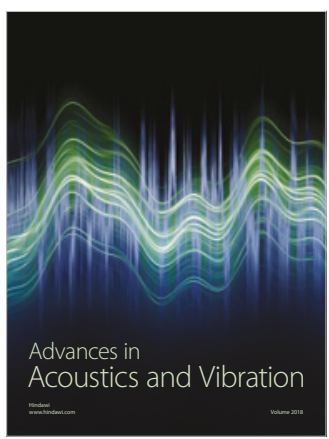

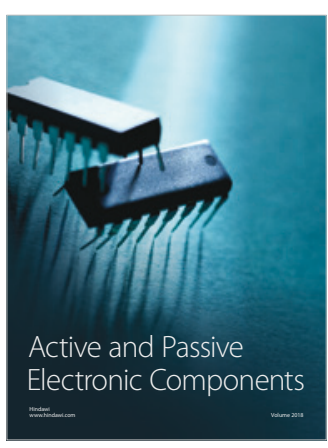
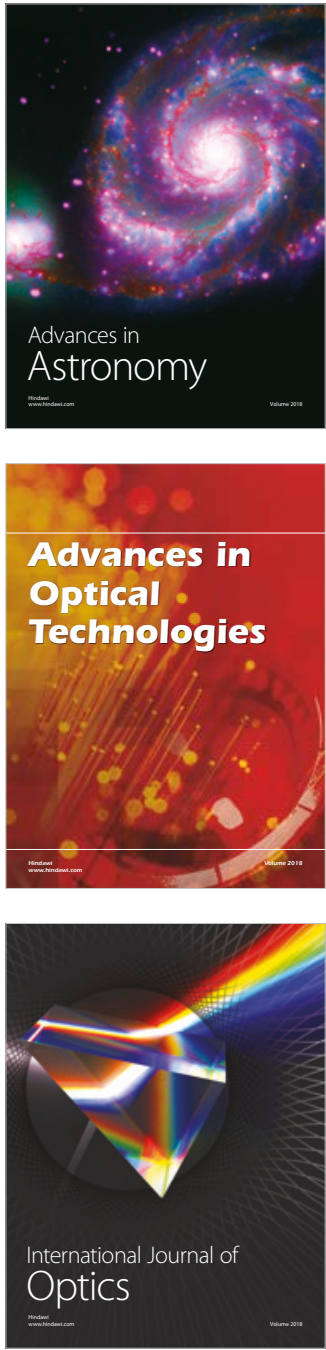